\title{
PERIODIC AUTOMORPHISMS, COMPATIBLE POISSON BRACKETS, AND GAUDIN SUBALGEBRAS
}

\author{
DMITRI I. PANYUSHEV* \\ Institute for \\ Information Transmission Problems \\ R.A.S. \\ Moscow 127051, Russia \\ panyushev@iitp.ru
}

\author{
OKSANA S. YAKIMOVA** \\ Institut für \\ Mathematik \\ Friedrich-Schiller-Universität Jena \\ 07737 Jena, Deutschland \\ oksana.yakimova@uni-jena.de
}

To the memory of our teacher Ernest Borisovich Vinberg

\begin{abstract}
Let $\mathfrak{q}$ be a finite-dimensional Lie algebra. The symmetric algebra $\mathcal{S}(\mathfrak{q})$ is equipped with the standard Lie-Poisson bracket. In this paper, we elaborate on a surprising observation that one naturally associates the second compatible Poisson bracket on $\mathcal{S}(\mathfrak{q})$ to any finite order automorphism $\vartheta$ of $\mathfrak{q}$. We study related Poisson-commutative subalgebras $\mathcal{Z}(\mathfrak{q}, \vartheta)$ of $\mathcal{S}(\mathfrak{q})$ and associated Lie algebra contractions of $\mathfrak{q}$. To obtain substantial results, we have to assume that $\mathfrak{q}=\mathfrak{g}$ is semisimple. Then we can use Vinberg's theory of $\vartheta$-groups and the machinery of Invariant Theory.

If $\mathfrak{g}=\mathfrak{h} \oplus \cdots \oplus \mathfrak{h}$ (sum of $k$ copies), where $\mathfrak{h}$ is simple, and $\vartheta$ is the cyclic permutation, then we prove that the corresponding Poisson-commutative subalgebra $z(\mathfrak{q}, \vartheta)$ is polynomial and maximal. Furthermore, we quantise this $z(\mathfrak{g}, \vartheta)$ using a Gaudin subalgebra in the enveloping algebra $\mathcal{U}(\mathfrak{g})$.
\end{abstract}

\section{Introduction}

The ground field $\mathbb{k}$ is algebraically closed and $\operatorname{char}(\mathbb{k})=0$. Let $\mathfrak{q}=(\mathfrak{q},[]$,$) be$ a finite-dimensional algebraic Lie algebra, i.e., $\mathfrak{q}=\operatorname{Lie} Q$, where $Q$ is a connected affine algebraic group. The dual space $\mathfrak{q}^{*}$ is a Poisson variety, i.e., the algebra of polynomial functions on $\mathfrak{q}^{*}, \mathbb{k}\left[\mathfrak{q}^{*}\right] \simeq \mathcal{S}(\mathfrak{q})$, is equipped with the Lie-Poisson bracket $\{$,$\} . Here \{x, y\}=[x, y]$ for $x, y \in \mathfrak{q}$. Poisson-commutative subalgebras of $\mathbb{k}\left[\mathfrak{q}^{*}\right]$ are important tools for the study of geometry of the coadjoint representation of $Q$ and representation theory of $\mathfrak{q}$.

DOI: $10.1007 / \mathrm{s} 00031-021-09650-3$

* The first author is funded by RFBR, project number 20-01-00515.

***Funded by the DFG (German Research Foundation) — project number 404144169.

Received September 1, 2020. Accepted February 24, 2021.

Published online April 6, 2021.

Corresponding Author: Oksana S. Yakimova, e-mail: oksana.yakimova@uni-jena.de 


\subsection{Compatible Poisson brackets}

There is a well-known method, the Lenard-Magri scheme, for constructing "large" Poisson-commutative subalgebras of $\mathbb{k}\left[\mathfrak{q}^{*}\right]$, which is related to compatible Poisson brackets, see, e.g., [GZ00]. Two Poisson brackets $\{,\}^{\prime}$ and $\{,\}^{\prime \prime}$ are said to be compatible, if any linear combination $\{,\}_{a, b}:=a\{,\}^{\prime}+b\{,\}^{\prime \prime}$ with $a, b \in \mathbb{k}$ is a Poisson bracket. Then one defines a certain dense open subset $\Omega_{\text {reg }} \subset \mathbb{k}^{2}$ that corresponds to the regular brackets in the pencil $\mathcal{P}=\left\{\{,\}_{a, b} \mid(a, b) \in \mathbb{k}^{2}\right\}$. If $\mathcal{Z}_{a, b} \subset \mathcal{S}(\mathfrak{q})$ denotes the Poisson centre of $\left(\mathcal{S}(\mathfrak{q}),\{,\}_{a, b}\right)$, then the subalgebra $\mathcal{Z} \subset \mathcal{S}(\mathfrak{q})$ generated by $\mathcal{Z}_{a, b}$ with $(a, b) \in \Omega_{\text {reg }}$ is Poisson-commutative with respect to $\{,\}^{\prime}$ and $\{,\}^{\prime \prime}$, see Section 2.2 for more details. An obvious first step is to take the initial Lie-Poisson bracket $\{$,$\} as \{,\}^{\prime}$. The rest depends on a clever choice of $\{,\}^{\prime \prime}$. The goal of this article is to introduce a new class of compatible Poisson brackets, study the respective subalgebras $Z$, and provide applications.

Let us recall some known pencils of compatible Poisson brackets.

- For any $\gamma \in \mathfrak{q}^{*}$, the Poisson bracket $(x, y) \mapsto\{x, y\}_{\gamma}:=\gamma([x, y]), x, y \in \mathfrak{q}$, is compatible with $\{$,$\} , cf. [DZ05, Example 1.8.16]. This leads to the fabulous$ Mishchenko-Fomenko $(=M F)$ subalgebras $\mathcal{Z}=\mathcal{A}_{\gamma} \subset \mathcal{S}(\mathfrak{q})$, introduced in [MF78] for semisimple $\mathfrak{q}$. To the best of our knowledge, the very term "MishchenkoFomenko" was coined by Vinberg [Vi90].

- In [PY], we presented compatible Poisson brackets related to a $\mathbb{Z}_{2}$-grading $\mathfrak{q}=\mathfrak{q}_{0} \oplus \mathfrak{q}_{1}$ and studied the respective subalgebra $z=z\left(\mathfrak{q}, \mathfrak{q}_{0}\right)$. Here the second bracket is defined by the relations $\{x, y\}^{\prime \prime}=\{x, y\}$ if $x \in \mathfrak{q}_{0}$ and $\{x, y\}^{\prime \prime}=0$ if $x, y \in \mathfrak{q}_{1}$. As well as with MF subalgebras, one cannot get too far if $\mathfrak{q}$ is arbitrary. Assuming that $\mathfrak{q}=\mathfrak{g}$ is reductive, we obtained a number of interesting results on $\mathcal{Z}\left(\mathfrak{g}, \mathfrak{g}_{0}\right)$. We proved that:

- $Z\left(\mathfrak{g}, \mathfrak{g}_{0}\right)$ is a Poisson-commutative subalgebra of $\mathcal{S}(\mathfrak{g})^{\mathfrak{g}_{0}}$ having the maximal possible transcendence degree, which equals $\left(\operatorname{dim} \mathfrak{g}_{1}+\operatorname{rk} \mathfrak{g}+\operatorname{rk} \mathfrak{g}_{0}\right) / 2$;

- with only four exceptions related to exceptional Lie algebras, $z\left(\mathfrak{g}, \mathfrak{g}_{0}\right)$ is a polynomial algebra whose algebraically independent generators are explicitly described;

— if $\mathfrak{g}$ is a classical Lie algebra and $\mathfrak{g}_{0}$ contains a regular nilpotent element of $\mathfrak{g}$, then $\mathcal{Z}\left(\mathfrak{g}, \mathfrak{g}_{0}\right)$ is a maximal Poisson-commutative subalgebra of $\mathcal{S}(\mathfrak{g})^{\mathfrak{g}_{0}}$.

The proofs exploit numerous invariant-theoretic properties of the adjoint representation of $\mathfrak{g}[\mathrm{K} 63]$ as well as their analogues for the isotropy representation $G_{0} \rightarrow \mathrm{GL}\left(\mathfrak{g}_{1}\right)[\mathrm{KR} 71]$.

\section{2. $\vartheta$-groups}

Results of the present paper stem from a surprising observation that if $\mathfrak{q}$ is equipped with a $\mathbb{Z}_{m}$-grading with any $m \geqslant 2$, then one can naturally construct a compatible Poisson bracket $\{,\}^{\prime \prime}$ (Section 2). In this case, all Poisson brackets in $\mathcal{P}$ are linear and there are two lines $l_{1}, l_{2} \subset \mathbb{k}^{2}$ such that $\Omega=\mathbb{k}^{2} \backslash\left(l_{1} \cup l_{2}\right) \subset \Omega_{\text {reg }}$ and the Lie algebras corresponding to $(a, b) \in \Omega$ are isomorphic to $\mathfrak{q}$. The lines $l_{1}$ and $l_{2}$ give rise to new Lie algebras, denoted $\mathfrak{q}_{(0)}$ and $\mathfrak{q}_{(\infty)}$. These new algebras are different contractions of $\mathfrak{q}$. Let ind $\mathfrak{q}$ denote the index of $\mathfrak{q}$ (see Section 1). Then ind $\mathfrak{q} \leqslant$ ind $\mathfrak{q}_{(n)}, n \in\{0, \infty\}$, and our first task is to realise whether it is true that ind $\mathfrak{q}=$ ind $\mathfrak{q}_{(n)}$. Although basic theory can be developed for arbitrary $\mathfrak{q}$, essential 
applications require a better class of Lie algebras, and we eventually stick to the semisimple case. Let $\vartheta \in \operatorname{Aut}(\mathfrak{g})$ be an automorphism of order $m$ and $\mathfrak{g}=\bigoplus_{j=0}^{m-1} \mathfrak{g}_{j}$ the associated $\mathbb{Z}_{m}$-grading, i.e., if $\zeta=\sqrt[m]{1}$ is primitive, then

$$
\mathfrak{g}_{j}=\left\{x \in \mathfrak{g} \mid \vartheta(x)=\zeta^{j} x\right\}
$$

The invariant-theoretic base for our consideration is Vinberg's theory of " $\vartheta$-groups" (i.e., theory of orbits and invariants for representations of reductive groups related to the periodic automorphisms of $\mathfrak{g}$ ), see [Vi76], [Vi79].

A bad news is that, for $m \geqslant 3$, the invariant-theoretic picture related to $\vartheta$ and properties of $\mathfrak{g}_{(0)}$ become more complicated. For instance, if $m=2$, then $\mathfrak{g}_{(0)} \simeq \mathfrak{g}_{0} \ltimes \mathfrak{g}_{1}^{\mathrm{ab}}$ (semi-direct product) and it is known that here ind $\mathfrak{g}_{(0)}=$ ind $\mathfrak{g}=$ $\mathrm{rk} \mathfrak{g}[\mathrm{P} 07]$. For $m \geqslant 3$, the number ind $\mathfrak{g}_{(0)}$ remains mysterious. We suspect that it is equal to rk $\mathfrak{g}$ for any $\vartheta$. Other technical difficulties are discussed in Section 3.3. Nevertheless, we succeeded in computing ind $\mathfrak{g}_{(\infty)}$, see Theorem 3.2 , and can state that ind $\mathfrak{g}_{(\infty)}=\mathrm{rk} \mathfrak{g}$ if and only if $\mathfrak{g}_{0}$ is abelian.

A good news is that there are still many interesting cases (automorphisms $\vartheta$ ), where analogues of results of [PY] are valid and also some unexpected applications pop up. Write $Z(\mathfrak{g}, \vartheta)$ for the Poisson-commutative subalgebra associated with the pencil of compatible Poisson brackets related to $\vartheta$. Let $\mathcal{N}$ be the set of nilpotent elements of $\mathfrak{g}$. Suppose that $\vartheta$ has the following properties:

(1) $\mathfrak{g}_{1}$ contains a regular semisimple element of $\mathfrak{g}$;

(2) each irreducible component of $\mathfrak{g}_{1} \cap \mathcal{N}$ contains a regular nilpotent element of $\mathfrak{g}$.

Under these assumptions we prove that $\mathcal{Z}(\mathfrak{g}, \vartheta)$ has the same properties as the above algebra $\mathcal{Z}\left(\mathfrak{g}, \mathfrak{g}_{0}\right)$, see Sections 4,5 .

\subsection{Cyclic permutations and Gaudin algebras}

More good news is that there is a special case of $(\mathfrak{g}, \vartheta)$, when $\mathcal{Z}(\mathfrak{g}, \vartheta)$ is as good as possible and it has a nice quantisation. Namely, let $\mathfrak{h}$ be a non-abelian simple Lie algebra and $\mathfrak{g}=\mathfrak{h}^{m}$ be the direct sum of $m$ copies of $\mathfrak{h}$. If $\vartheta$ is the cyclic permutation of the summands, then $\mathfrak{g}_{0}=\Delta_{\mathfrak{h}} \simeq \mathfrak{h}$ and we prove in Section 6 that $\mathfrak{Z}=\mathfrak{Z}\left(\mathfrak{h}^{m}, \vartheta\right)$ is a polynomial ring in $\frac{1}{2}((m-1) \operatorname{dim} \mathfrak{h}+(m+1) \operatorname{rk} \mathfrak{h})$ generators. Furthermore, $\boldsymbol{Z}$ is a maximal Poisson-commutative subalgebra of $\mathcal{S}(\mathfrak{g})^{\mathfrak{g}_{0}}$. The quantisation problem asks for a lift of $\mathcal{Z}$ to the enveloping algebra $\mathcal{U}(\mathfrak{g})$, i.e., for a commutative algebra $\tilde{z} \subset \mathcal{U}(\mathfrak{g})$ such that $\operatorname{gr}(\tilde{z})=z$. To describe $\tilde{z}$ in this context, we need some preparations.

The enveloping algebra $\mathfrak{U}\left(\mathfrak{h}\left[t, t^{-1}\right]\right)$ of the loop algebra $\mathfrak{h}\left[t, t^{-1}\right]$ contains a large commutative subalgebra $\mathfrak{z}(\widehat{\mathfrak{h}})$ of infinite transcendence degree, known as the FeiginFrenkel centre $\left[\right.$ FF92]. Actually $\mathfrak{z}(\widehat{\mathfrak{h}}) \subset \mathcal{U}\left(\widehat{\mathfrak{h}}_{-}\right)$, where $\widehat{\mathfrak{h}}_{-}=t^{-1} \mathfrak{h}\left[t^{-1}\right]$. Having a vector $\vec{z} \in\left(\mathbb{k}^{\star}\right)^{m}$, one defines a homomorphism $\rho_{\vec{z}}: \mathcal{U}\left(\widehat{\mathfrak{h}}_{-}\right) \rightarrow \mathcal{U}(\mathfrak{g})$. The image of $\mathfrak{z}(\widehat{\mathfrak{h}})$ under $\rho_{\vec{z}}$ is called the Gaudin subalgebra $\mathcal{G}(\vec{z})$ [FFR]. For the case where the entries of $\vec{z}$ are pairwise distinct $m$-th roots of unity, we provide a simpler construction of $\mathcal{G}(\vec{z})$ (Proposition 7.2 ) and show that $\operatorname{gr}(\mathcal{G}(\vec{z}))=\mathcal{Z}\left(\mathfrak{h}^{m}, \vartheta\right)$, see Theorem 7.4. 
It is worth noting that, for the MF subalgebras $\mathcal{A}_{\gamma} \subset \mathcal{S}(\mathfrak{h})$, the quantisation problem was posed by Vinberg [Vi90]. A solution given by Rybnikov [R06] states that the image of $\mathfrak{z}(\widehat{\mathfrak{h}})$ under a certain homomorphism $\varrho_{\gamma}: \mathfrak{U}\left(\widehat{\mathfrak{h}}_{-}\right) \rightarrow \mathcal{U}(\mathfrak{h})$, depending on $\gamma \in \mathfrak{h}^{*}$, is the quantum MF subalgebra $\tilde{\mathcal{A}}_{\gamma}$ and one has $\operatorname{gr}\left(\tilde{\mathcal{A}}_{\gamma}\right)=\mathcal{A}_{\gamma}$ in many cases.

Let us identify $\widehat{\mathfrak{h}}_{-}$with the quotient space $\mathfrak{h}\left[t, t^{-1}\right] / \mathfrak{h}[t]$. Then $\mathfrak{h}[t]$ acts on $t^{-1} \mathfrak{h}\left[t^{-1}\right]$ and correspondingly on $\mathcal{S}\left(\widehat{\mathfrak{h}}_{-}\right)$. By [FF92], [F07], one has

$$
\operatorname{gr}(\mathfrak{z}(\widehat{\mathfrak{h}}))=\mathcal{S}\left(\widehat{\mathfrak{h}}_{-}\right)^{\mathfrak{h}[t]}
$$

Yet another property of the Poisson-commutative algebra $\operatorname{gr}(\mathfrak{z}(\widehat{\mathfrak{h}}))$ is that it is a polynomial ring in infinitely many variables, by a direct generalisations of a RaïsTauvel theorem [RT92].

Suppose that $\vartheta \in \operatorname{Aut}(\mathfrak{h})$. In Section 8, we consider the $\vartheta$-twisted version of $\operatorname{gr}(\mathfrak{z}(\widehat{\mathfrak{h}}))$, a certain subalgebra $\mathcal{Z}\left(\widehat{\mathfrak{h}}_{-}, \vartheta\right)$ of $\mathcal{S}\left(\left(\widehat{\mathfrak{h}}_{-}\right)^{\vartheta}\right)$ of infinite transcendence degree. Assuming the equality ind $\mathfrak{h}_{(0)}=\operatorname{rk} \mathfrak{h}$, we prove that $\mathfrak{Z}\left(\widehat{\mathfrak{h}}_{-}, \vartheta\right)$ is Poissoncommutative, see Theorem 8.2 . In many cases, $z\left(\widehat{\mathfrak{h}}_{-}, \vartheta\right)$ is a polynomial ring.

Our general reference for semisimple Lie groups and algebras is [Lie3].

\section{Preliminaries on Poisson brackets and polynomial contractions}

Let $Q$ be a connected affine algebraic group with Lie algebra $\mathfrak{q}$. The symmetric algebra of $\mathfrak{q}$ over $\mathbb{k}$ is $\mathbb{N}$-graded, i.e., $\mathcal{S}(\mathfrak{q})=\bigoplus_{i \geqslant 0} \mathcal{S}^{i}(\mathfrak{q})$. It is identified with the algebra of polynomial functions on the dual space $\mathfrak{q}^{*}$, and we also write $\mathbb{k}\left[\mathfrak{q}^{*}\right]=$ $\bigoplus_{i \geqslant 0} \mathbb{k}\left[\mathfrak{q}^{*}\right]_{i}$ for it.

\subsection{The coadjoint representation}

The group $Q$ acts on $\mathfrak{q}^{*}$ via the coadjoint representation and $\operatorname{ad}^{*}: \mathfrak{q} \rightarrow \operatorname{GL}\left(\mathfrak{q}^{*}\right)$ is the coadjoint representation of $\mathfrak{q}$. The algebra of $Q$-invariant polynomial functions on $\mathfrak{q}^{*}$ is denoted by $\mathcal{S}(\mathfrak{q})^{Q}$ or $\mathbb{k}\left[\mathfrak{q}^{*}\right]^{Q}$. Write $\mathbb{k}\left(\mathfrak{q}^{*}\right)^{Q}$ for the field of $Q$-invariant rational functions on $\mathfrak{q}^{*}$.

Let $\mathfrak{q}^{\xi}=\left\{x \in \mathfrak{q} \mid \operatorname{ad}^{*}(x) \cdot \xi=0\right\}$ be the stabiliser in $\mathfrak{q}$ of $\xi \in \mathfrak{q}^{*}$. The index of $\mathfrak{q}$, ind $\mathfrak{q}$, is the minimal codimension of $Q$-orbits in $\mathfrak{q}^{*}$. Equivalently,

$$
\text { ind } \mathfrak{q}=\min _{\xi \in \mathfrak{q}^{*}} \operatorname{dim} \mathfrak{q}^{\xi} \text {. }
$$

By the Rosenlicht theorem (see $\left[\operatorname{Sp} 89\right.$, IV.2]), one also has ind $\mathfrak{q}=\operatorname{tr} \cdot \operatorname{deg} \mathbb{k}\left(\mathfrak{q}^{*}\right)^{Q}$. Set $\boldsymbol{b}(\mathfrak{q})=(\operatorname{dim} \mathfrak{q}+$ ind $\mathfrak{q}) / 2$. Since the $Q$-orbits in $\mathfrak{q}^{*}$ are even-dimensional, $\boldsymbol{b}(\mathfrak{q})$ is an integer. If $\mathfrak{q}$ is reductive, then ind $\mathfrak{q}=\operatorname{rk} \mathfrak{q}$ and $\boldsymbol{b}(\mathfrak{q})$ equals the dimension of a Borel subalgebra.

The Lie-Poisson bracket in $\mathcal{S}(\mathfrak{q})$ is defined on $\mathcal{S}^{1}(\mathfrak{q})=\mathfrak{q}$ by $\{x, y\}:=[x, y]$. It is then extended to higher degrees via the Leibniz rule. Hence $\mathcal{S}(\mathfrak{q})$ has the usual associative-commutative structure and additional Poisson structure. Whenever we refer to subalgebras of $\mathcal{S}(\mathfrak{q})$, we always mean the associative-commutative structure. Then a subalgebra $\mathcal{A} \subset \mathcal{S}(\mathfrak{q})$ is said to be Poisson-commutative, if $\{H, F\}=0$ for all $H, F \in \mathcal{A}$. It is well known that if $\mathcal{A}$ is Poisson-commutative, 
then tr.deg $\mathcal{A} \leqslant \boldsymbol{b}(\mathfrak{q})$, see, e.g., [Vi90, 0.2]. More generally, suppose that $\mathfrak{h} \subset \mathfrak{q}$ is a Lie subalgebra and $\mathcal{A} \subset \mathcal{S}(\mathfrak{q})^{\mathfrak{h}}$ is Poisson-commutative. Then

$$
\operatorname{tr} . \operatorname{deg} \mathcal{A} \leqslant \boldsymbol{b}(\mathfrak{q})-\boldsymbol{b}(\mathfrak{h})+\operatorname{ind} \mathfrak{h},
$$

see [MY19, Prop. 1.1].

The centre of the Poisson algebra $(\mathcal{S}(\mathfrak{q}),\{\}$,$) is$

$$
\mathcal{Z}(\mathfrak{q}):=\{H \in \mathcal{S}(\mathfrak{q}) \mid\{H, F\}=0 \quad \forall F \in \mathcal{S}(\mathfrak{q})\}
$$

Using the Leibniz rule, we obtain that $\mathcal{Z}(\mathfrak{q})$ is a graded Poisson-commutative subalgebra of $\mathcal{S}(\mathfrak{q})$, which coincides with the algebra of symmetric invariants of $\mathfrak{q}$, i.e.,

$$
\mathcal{Z}(\mathfrak{q})=\{H \in \mathcal{S}(\mathfrak{q}) \mid\{H, x\}=0 \quad \forall x \in \mathfrak{q}\}=: \mathcal{S}(\mathfrak{q})^{\mathfrak{q}}=\mathbb{k}\left[\mathfrak{q}^{*}\right]^{\mathfrak{q}}
$$

As $Q$ is connected, we have $\mathcal{S}(\mathfrak{q})^{\mathfrak{q}}=\mathcal{S}(\mathfrak{q})^{Q}=\mathbb{k}\left[\mathfrak{q}^{*}\right]^{Q}$. Since the quotient field of $\mathbb{k}\left[\mathfrak{q}^{*}\right]^{Q}$ is contained in $\mathbb{k}\left(\mathfrak{q}^{*}\right)^{Q}$, we deduce from the Rosenlicht theorem that

$$
\operatorname{tr} \cdot \operatorname{deg}\left(\mathcal{S}(\mathfrak{q})^{\mathfrak{q}}\right) \leqslant \operatorname{ind} \mathfrak{q} \text {. }
$$

The set of $Q$-regular elements of $\mathfrak{q}^{*}$ is

$$
\mathfrak{q}_{\text {reg }}^{*}=\left\{\eta \in \mathfrak{q}^{*} \mid \operatorname{dim} \mathfrak{q}^{\eta}=\operatorname{ind} \mathfrak{q}\right\}=\left\{\eta \in \mathfrak{q}^{*} \mid \operatorname{dim} Q \cdot \eta \text { is maximal }\right\} .
$$

It is a dense open subset of $\mathfrak{q}^{*}$. Set $\mathfrak{q}_{\text {sing }}^{*}=\mathfrak{q}^{*} \backslash \mathfrak{q}_{\text {reg }}^{*}$. We say that $\mathfrak{q}$ has the codim $-n$ property if codim $\mathfrak{q}_{\text {sing }}^{*} \geqslant n$. The codim-2 property is going to be most important for us.

For $\gamma \in \mathfrak{q}^{*}$, let $\hat{\gamma}$ be the skew-symmetric bilinear form on $\mathfrak{q}$ defined by $\hat{\gamma}(\xi, \eta)=$ $\gamma([\xi, \eta])$ for $\xi, \eta \in \mathfrak{q}$. It follows that $\operatorname{ker} \hat{\gamma}=\mathfrak{q}^{\gamma}$. The 2 -form $\hat{\gamma}$ is related to the Poisson tensor (bivector) $\pi$ of the Lie-Poisson bracket $\{$,$\} as follows.$

Let $d H$ denote the differential of $H \in \mathcal{S}(\mathfrak{q})=\mathbb{k}\left[\mathfrak{q}^{*}\right]$. Then $\pi$ is defined by the formula

$$
\pi(d H \wedge d F)=\{H, F\} \text { for } H, F \in \mathcal{S}(\mathfrak{q}) .
$$

Here $\pi(\gamma)\left(d_{\gamma} H \wedge d_{\gamma} F\right)=\{H, F\}(\gamma)$ and therefore $\hat{\gamma}=\pi(\gamma)$. In this term, ind $\mathfrak{q}=$ $\operatorname{dim} \mathfrak{q}-\operatorname{rk} \pi$, where $\operatorname{rk} \pi=\max _{\gamma \in \mathfrak{q}^{*}} \operatorname{rk} \pi(\gamma)$.

\subsection{Contractions and invariants}

We refer to [Lie3, Chap. $7, \S 2]$ for basic facts on contractions of Lie algebras. In this article, we consider contractions of the following form. Let $\mathbb{k}^{\star}=\mathbb{k} \backslash\{0\}$ be the multiplicative group of $\mathbb{k}$ and $\varphi: \mathbb{k}^{\star} \rightarrow \operatorname{GL}(\mathfrak{q}), s \mapsto \varphi_{s}$, a polynomial representation. That is, the matrix entries of $\varphi_{s}: \mathfrak{q} \rightarrow \mathfrak{q}$ are polynomials in $s$ with respect to some (any) basis of $\mathfrak{q}$. Define a new Lie algebra structure on the vector space $\mathfrak{q}$ and associated Lie-Poisson bracket by

$$
[x, y]_{(s)}=\{x, y\}_{(s)}:=\varphi_{s}^{-1}\left[\varphi_{s}(x), \varphi_{s}(y)\right], x, y \in \mathfrak{q}, s \in \mathbb{k}^{\star}
$$

The corresponding Lie algebra is denoted by $\mathfrak{q}_{(s)}$. Then $\mathfrak{q}_{(1)}=\mathfrak{q}$ and all these algebras are isomorphic. The induced $\mathbb{k}^{\star}$-action in the variety of structure constants 
in not necessarily polynomial, i.e., $\lim _{s \rightarrow 0}[x, y]_{(s)}$ may not exist for all $x, y \in \mathfrak{q}$. Whenever such a limit exists, we obtain a new linear Poisson bracket, denoted $\{,\}_{0}$, and thereby a new Lie algebra $\mathfrak{q}_{(0)}$, which is said to be a contraction of $\mathfrak{q}$. If we wish to stress that this construction is determined by $\varphi$, then we write $\{x, y\}_{(\varphi, s)}$ for the bracket in $(1 \cdot 3)$ and say that $\mathfrak{q}_{(0)}$ is the $\varphi$-contraction of $\mathfrak{q}$ or is the zero limit of $\mathfrak{q}$ with respect to $\varphi$. A criterion for the existence of $\mathfrak{q}_{(0)}$ can be given in terms of Lie brackets of the $\varphi$-eigenspaces in $\mathfrak{q}$, see [Y17, Sect.4]. We identify all algebras $\mathfrak{q}_{(s)}$ and $\mathfrak{q}_{(0)}$ as vector spaces. The semi-continuity of index implies that ind $\mathfrak{q}_{(0)} \geqslant$ ind $\mathfrak{q}$.

The map $\varphi_{s}, s \in \mathbb{k}^{\star}$, is naturally extended to an invertible transformation of $\mathcal{S}^{j}(\mathfrak{q})$, which we also denote by $\varphi_{s}$. The resulting graded map $\varphi_{s}: \mathcal{S}(\mathfrak{q}) \rightarrow \mathcal{S}(\mathfrak{q})$ is nothing but the comorphism associated with $s \in \mathbb{k}^{\star}$ and the dual representation $\varphi^{*}: \mathbb{k}^{\star} \rightarrow \mathrm{GL}\left(\mathfrak{q}^{*}\right)$. Since $\mathcal{S}^{j}(\mathfrak{q})$ has a basis that consists of $\varphi\left(\mathbb{k}^{\star}\right)$-eigenvectors, any $F \in \mathcal{S}^{j}(\mathfrak{q})$ can be written as $F=\sum_{i \geqslant 0} F_{i}$, where the sum is finite and $\varphi_{s}\left(F_{i}\right)=s^{i} F_{i} \in \mathcal{S}^{j}(\mathfrak{q})$. Let $F^{\bullet}$ denote the nonzero component $F_{i}$ with maximal $i$.

Proposition 1.1 ([Y14, Lem. 3.3]). If $F \in \mathcal{Z}(\mathfrak{q})$ and $\mathfrak{q}_{(0)}$ exists, then $F^{\bullet} \in \mathcal{Z}\left(\mathfrak{q}_{(0)}\right)$.

\section{Automorphisms of finite order and compatible Poisson brackets}

In this section, we associate a pencil of compatible Poisson brackets to any automorphism of finite order of a Lie algebra $\mathfrak{q}$, describe the limit algebras $\mathfrak{q}_{(0)}$ and $\mathfrak{q}_{(\infty)}$, and construct the related Poisson-commutative subalgebra of $\mathcal{S}(\mathfrak{q})$.

\subsection{Periodic gradings of Lie algebras}

Let $\vartheta \in \operatorname{Aut}(\mathfrak{q})$ be a Lie algebra automorphism of finite order $m \geqslant 2$ and $\zeta=\sqrt[m]{1}$ a primitive root of unity. Write also $\operatorname{ord}(\vartheta)$ for the order of $\vartheta$. If $\mathfrak{q}_{i}$ is the $\zeta^{i}-$ eigenspace of $\vartheta, i \in \mathbb{Z}_{m}$, then the direct sum $\mathfrak{q}=\bigoplus_{i \in \mathbb{Z}_{m}} \mathfrak{q}_{i}$ is a periodic grading or $\mathbb{Z}_{m^{-}}$grading of $\mathfrak{q}$. The latter means that $\left[\mathfrak{q}_{i}, \mathfrak{q}_{j}\right] \subset \mathfrak{q}_{i+j}$ for all $i, j \in \mathbb{Z}_{m}$. Here $\mathfrak{q}_{0}=\mathfrak{q}^{\vartheta}$ is the fixed-point subalgebra for $\vartheta$ and each $\mathfrak{q}_{i}$ is a $\mathfrak{q}_{0}$-module. We will be primarily interested in periodic gradings of semisimple Lie algebras, but such a general setting is going to be useful, too.

We choose $\{0,1, \ldots, m-1\} \subset \mathbb{Z}$ as a fixed set of representatives for $\mathbb{Z}_{m}=\mathbb{Z} / m \mathbb{Z}$. Under this convention, we have $\mathfrak{q}=\mathfrak{q}_{0} \oplus \mathfrak{q}_{1} \oplus \ldots \oplus \mathfrak{q}_{m-1}$ and

$$
\left[\mathfrak{q}_{i}, \mathfrak{q}_{j}\right] \subset \begin{cases}\mathfrak{q}_{i+j}, & \text { if } i+j \leqslant m-1, \\ \mathfrak{q}_{i+j-m}, & \text { if } i+j \geqslant m .\end{cases}
$$

This is needed below, when we consider $\mathbb{Z}$-graded contractions of $\mathfrak{q}$ associated with $\vartheta$.

The presence of $\vartheta$ allows us to split the Lie-Poisson bracket on $\mathfrak{q}^{*}$ into a sum of two compatible linear Poisson brackets, as follows. Consider the polynomial representation $\varphi: \mathbb{k}^{\star} \rightarrow \operatorname{GL}(\mathfrak{q})$ such that $\varphi_{s}(x)=s^{j} x$ for $x \in \mathfrak{q}_{j}$. As in Section 1.2, this defines a family of linear Poisson brackets in $\mathcal{S}(\mathfrak{q})$ parametrised by $s \in \mathbb{k}^{\star}$, see $(1 \cdot 3)$. 
Proposition 2.1. For any $\vartheta \in \operatorname{Aut}(\mathfrak{q})$ of finite order $m$ and $\varphi$ as above, we have

(i) The map $\varphi_{s}^{-1}: \mathfrak{q} \rightarrow \mathfrak{q}$ provides an isomorphism between $(\mathfrak{q},[]$,$) and$ $\left(\mathfrak{q},[,]_{(s)}\right)$. In particular, the Poisson brackets $\{,\}_{(s)}, s \in \mathbb{K}^{\star}$, are isomorphic;

(ii) there is a limit $\lim _{s \rightarrow 0}\{x, y\}_{(s)}=:\{x, y\}_{0}$, which is a linear Poisson bracket in $\mathcal{S}(\mathfrak{q})$;

(iii) the difference $\{\}-,\{,\}_{0}=:\{,\}_{\infty}$ is a linear Poisson bracket on $\mathcal{S}(\mathfrak{q})$ and

$$
\{,\}_{(s)}=\{,\}_{0}+s^{m}\{,\}_{\infty} \text { for any } s \in \mathbb{k}^{\star} .
$$

(iv) The Poisson bracket $\{,\}_{\infty}$ is obtained as the zero limit with respect to the polynomial representation $\psi: \mathbb{k}^{\star} \rightarrow \mathrm{GL}(\mathfrak{q})$ such that $\psi_{s}=s^{m} \cdot \varphi_{s^{-1}}=$ $s^{m} \cdot \varphi_{s}^{-1}, s \in \mathbb{K}^{\star}$. In other words, we have $\{,\}_{\infty}=\lim _{s \rightarrow 0}\{,\}_{(\psi, s)}$.

Proof. (i) This readily follows from Eq. (1·3).

(ii) If $x \in \mathfrak{q}_{i}$ and $y \in \mathfrak{q}_{j}$, then

$$
\{x, y\}_{(s)}= \begin{cases}{[x, y],} & \text { if } i+j<m \\ s^{m}[x, y], & \text { if } i+j \geqslant m .\end{cases}
$$

Therefore, the limit of $\{x, y\}_{(s)}$ as $s$ tends to zero exists and is given by

$$
\{x, y\}_{0}:= \begin{cases}{[x, y],} & \text { if } i+j<m \\ 0, & \text { if } i+j \geqslant m\end{cases}
$$

The limit of Poisson brackets is again a Poisson bracket, hence the Jacobi identity is satisfied for $\{,\}_{0}$, cf. Section 1.2. However, this is easily verified directly.

(iii) By the above formula for $\{x, y\}_{0}$, we have

$$
\{x, y\}_{\infty}= \begin{cases}0, & \text { if } i+j<m \\ {[x, y],} & \text { if } i+j \geqslant m\end{cases}
$$

Therefore $\{x, y\}_{(s)}=\{x, y\}_{0}+s^{m}\{x, y\}_{\infty}$ for all $x, y \in \mathfrak{q}$. It is also easily verified that $\{,\}_{\infty}$ satisfies the Jacobi identity.

(iv) We have $\psi_{s}(x)=s^{m-i} x$ for $x \in \mathfrak{q}_{i}$. Then an easy calculation shows that the $(\psi, s)$-bracket is given by

$$
\{x, y\}_{(\psi, s)}= \begin{cases}s^{m}[x, y], & \text { if } i+j<m \\ {[x, y],} & \text { if } i+j \geqslant m\end{cases}
$$

Two Poisson brackets on the algebra $\mathcal{S}(\mathfrak{q})$ are said to be compatible, if any linear combination of them is again a Poisson bracket. Actually, if $\{,\}_{1}$ and $\{,\}_{2}$ are Poisson brackets, then it suffices to check that just $\{,\}_{1}+\{,\}_{2}$ is a Poisson bracket [PY, Lem. 1.1]. Anyway, we have 
Corollary 2.2. For any $\vartheta \in$ Aut $(\mathfrak{q})$ of finite order, the Poisson brackets $\{,\}_{0}$ and $\{,\}_{\infty}$ are compatible, and the corresponding pencil contains the initial LiePoisson bracket.

All Poisson brackets involved in Proposition 2.1 are linear. Therefore, in place of Poisson brackets on the symmetric algebra $\mathcal{S}(\mathfrak{q})$, we can stick to the corresponding Lie algebra structures on the vector space $\mathfrak{q}$. Let $\mathfrak{q}_{(s)}$ be the Lie algebra corresponding to $\{,\}_{(s)}$. Then all algebras with $s \in \mathbb{k}^{\star}$ are isomorphic, whereas the brackets $\{,\}_{0}$ and $\{,\}_{\infty}$ give rise to entirely different Lie algebras $\mathfrak{q}_{(0)}$ and $\mathfrak{q}_{(\infty)}$, respectively. Both $\mathfrak{q}_{(0)}$ and $\mathfrak{q}_{(\infty)}$ are Lie algebra contractions of $\mathfrak{q}$ in the sense of [Lie3, Chap. 7, §2]. Therefore ind $\mathfrak{q}_{(0)} \geqslant$ ind $\mathfrak{q}$ and ind $\mathfrak{q}_{(\infty)} \geqslant$ ind $\mathfrak{q}$ (the semi-continuity of the index).

Proposition 2.3. The Lie algebras $\mathfrak{q}_{(0)}$ and $\mathfrak{q}_{(\infty)}$ are $\mathbb{N}$-graded. More precisely, if $\mathfrak{r}[i]$ stands for the component of grade $i \in \mathbb{N}$ in an $\mathbb{N}$-graded Lie algebra $\mathfrak{r}$, then

$$
\mathfrak{q}_{(0)}[i]=\left\{\begin{array}{ll}
\mathfrak{q}_{i} & \text { for } i=0,1, \ldots, m-1, \\
0 & \text { otherwise }
\end{array} \quad \mathfrak{q}_{(\infty)}[i]= \begin{cases}\mathfrak{q}_{m-i} & \text { for } i=1,2, \ldots, m \\
0 & \text { otherwise }\end{cases}\right.
$$

In particular, $\mathfrak{q}_{(\infty)}$ is nilpotent and the subspace $\mathfrak{q}_{0}$, which is the highest grade component of $\mathfrak{q}_{(\infty)}$, belongs to the centre of $\mathfrak{q}_{(\infty)}$.

Proof. Use the formulae for $\{x, y\}_{0}$ and $\{x, y\}_{\infty}$ from the proof of Proposition 2.1.

\subsection{Poisson-commutative subalgebras related to compatible Poisson brackets}

There is a general method for constructing a Poisson-commutative subalgebra of $\mathcal{S}(\mathfrak{q})$ with "large" transcendence degree that exploits compatible Poisson brackets and the centre of $\mathcal{S}(\mathfrak{q})$, see, e.g., [DZ05, Sect. 1.8.3], [GZ00, Sect. 10], [PY, Sect. 2]. We recall this method in our present setting.

By Proposition 2.1, we have

$$
\{,\}=\{,\}_{0}+\{,\}_{\infty} \quad \text { and } \quad\{,\}_{(s)}=\{,\}_{0}+s^{m}\{,\}_{\infty} .
$$

Since $\{,\}_{(s)}=\{,\}_{\left(s^{\prime}\right)}$ if $s^{m}=\left(s^{\prime}\right)^{m}$, it is convenient to replace $s^{m}$ with $t$ and set

$$
\{,\}_{t}=\{,\}_{0}+t\{,\}_{\infty},
$$

where $t \in \mathbb{P}:=\mathbb{k} \cup\{\infty\}$ and the value $t=\infty$ corresponds to the bracket $\{,\}_{\infty}$. We will use the parameter $s \in \mathbb{k}^{\star}$, when the multiplicative group $\varphi: \mathbb{k}^{\star} \rightarrow \operatorname{GL}(\mathfrak{q})$ is needed.

Let $\mathfrak{q}_{(t)}$ stand for the Lie algebra corresponding to $\{,\}_{t}$. All these Lie algebras have the same underlying vector space. Since the algebras $\mathfrak{q}_{(t)}$ with $t \in \mathbb{k}^{\star}$ are isomorphic, they have one and the same index. We say that $t \in \mathbb{P}$ is regular if ind $\mathfrak{q}_{(t)}=$ ind $\mathfrak{q}$ and let $\mathbb{P}_{\text {reg }}$ stand for the set of regular values. Then $\mathbb{P}_{\text {sing }}:=$ $\mathbb{P} \backslash \mathbb{P}_{\text {reg }} \subset\{0, \infty\}$ is the set of singular values.

Let $\mathcal{Z}_{t}$ be the centre of the Poisson algebra $\left(\mathcal{S}(\mathfrak{q}),\{,\}_{t}\right)$. In particular, $\mathcal{Z}_{1}=$ $\mathcal{S}(\mathfrak{q})^{\mathfrak{q}}$. If $t \in \mathbb{k}^{\star}$, then $\mathcal{Z}_{t}=\varphi_{s}^{-1}\left(\mathcal{Z}_{1}\right)$, where $s^{m}=t$. By Eq. (1.1), we have 
tr.deg $\mathcal{Z}_{t} \leqslant$ ind $\mathfrak{q}_{(t)}$. Our main object is the subalgebra $z \subset \mathcal{S}(\mathfrak{q})$ generated by the centres $\mathcal{Z}_{t}$ with $t \in \mathbb{P}_{\text {reg }}$, i.e.,

$$
z=z(\mathfrak{q}, \vartheta)=\operatorname{alg}\left\langle\mathcal{Z}_{t} \mid t \in \mathbb{P}_{\text {reg }}\right\rangle
$$

By a general property of compatible brackets, the algebra $Z$ is Poisson-commutative with respect to all brackets $\{,\}_{t}$ with $t \in \mathbb{P}$, cf. [PY, Sect. 2]. Note that the Lie subalgebra $\mathfrak{q}_{0} \subset \mathfrak{q}=\mathfrak{q}_{(1)}$ is also the same Lie subalgebra in any $\mathfrak{q}_{(t)}$ with $t \neq \infty$ (cf. Proposition 2.3 for $\left.\mathfrak{q}_{(0)}\right)$. Therefore,

$$
\mathcal{Z}_{t} \subset \mathcal{S}(\mathfrak{q})^{\mathfrak{q}_{0}} \text { for } t \neq \infty
$$

In general, one cannot say much about $z \subset \mathcal{S}(\mathfrak{q})$. To arrive at more definite conclusions on $z$, a lot of extra information on $\mathcal{S}(\mathfrak{q})^{\mathfrak{q}}, \mathfrak{q}_{(0)}$, and $\mathfrak{q}_{(\infty)}$ is required. In particular, one has to know whether 0 and/or $\infty$ belong to $\mathbb{P}_{\text {reg. }}$. And this is the reason why we have to stick to semisimple Lie algebras.

\section{Poisson-commutative subalgebras of $\mathcal{S}(\mathfrak{g})$ : the semisimple case}

From now on, $G$ is a connected semisimple algebraic group and $\mathfrak{g}=$ Lie $G$. We consider $\vartheta \in \operatorname{Aut}(\mathfrak{g})$ of order $m \geqslant 2$ and freely use the previous notation and results, with $\mathfrak{q}$ being replaced by $\mathfrak{g}$. In particular,

$$
\mathfrak{g}=\mathfrak{g}_{0} \oplus \mathfrak{g}_{1} \oplus \ldots \oplus \mathfrak{g}_{m-1}
$$

where $\{0,1, \ldots, m-1\}$ is the fixed set of representatives for $\mathbb{Z}_{m}$, and $G_{0}$ is the connected subgroup of $G$ with Lie $\left(G_{0}\right)=\mathfrak{g}_{0}$. Then $\mathfrak{g}_{(t)}$ is a family of Lie algebras parameterised by $t \in \mathbb{P}=\mathbb{k} \cup\{\infty\}$, where the algebras $\mathfrak{g}_{(t)}$ with $t \in \mathbb{k}^{\star}$ are isomorphic to $\mathfrak{g}=\mathfrak{g}_{(1)}$, while $\mathfrak{g}_{(0)}$ and $\mathfrak{g}_{(\infty)}$ are different $\mathbb{N}$-graded contractions of $\mathfrak{g}$. Next, $\mathcal{Z}_{t}$ is the Poisson centre of $\left(\mathcal{S}(\mathfrak{g}),\{,\}_{t}\right)$ and the construction of Section 2.2 provides a Poisson-commutative subalgebra $\mathcal{Z}=\operatorname{alg}\left\langle\mathcal{Z}_{t} \mid t \in \mathbb{P}_{\text {reg }}\right\rangle \subset \mathcal{S}(\mathfrak{g})$. The connected algebraic group corresponding to $\mathfrak{g}_{(t)}$ is denoted by $G_{(t)}$.

Our goal is to demonstrate that there are many interesting cases, in which $z$ is a polynomial algebra having the maximal possible transcendence degree. Let us recall standard invariant-theoretic properties of semisimple Lie algebras.

The Poisson centre $\mathcal{S}(\mathfrak{g})^{\mathfrak{g}}=\mathfrak{S}(\mathfrak{g})^{G}$ is a polynomial algebra of Krull dimension $l=\operatorname{rk} \mathfrak{g}$ and ind $\mathfrak{g}=l$. Hence one has now the equality in Eq. (1·1). Note also that $\mathfrak{g}_{0}$ is a reductive Lie algebra. Write $\mathcal{N}$ for the cone of nilpotent elements of $\mathfrak{g}$. Let $\varkappa$ be the Killing form on $\mathfrak{g}$. We identify $\mathfrak{g}$ and $\mathfrak{g}_{0}$ with their duals via $\varkappa$. Moreover, since $\varkappa\left(\mathfrak{g}_{i}, \mathfrak{g}_{j}\right)=0$ if $i+j \notin\{0, m\}$, the dual space of $\mathfrak{g}_{j}, \mathfrak{g}_{j}^{*}$, can be identified with $\mathfrak{g}_{m-j}$. Here $\mathfrak{g}_{\text {reg }}^{*}=\mathfrak{g}_{\text {reg }}=\left\{x \in \mathfrak{g} \mid \operatorname{dim} \mathfrak{g}^{x}=l\right\}$. By [K63], $\mathfrak{g}$ has the codim-3 property, i.e., $\operatorname{codim}_{\mathfrak{g}}\left(\mathfrak{g} \backslash \mathfrak{g}_{\text {reg }}\right)=3$. Recall also that $\mathcal{N} \cap \mathfrak{g}_{\text {reg }}$ is non-empty, and it is a sole $G$-orbit, the regular nilpotent orbit.

Convention. We think of $\mathfrak{g}^{*}$ as the dual space for any Lie algebra $\mathfrak{g}_{(t)}$ and sometimes omit the subscript ' $(t)^{\prime}$ ' in $\mathfrak{g}_{(t)}^{*}$. However, if $\xi \in \mathfrak{g}^{*}$, then the stabiliser of $\xi$ with respect to the coadjoint representation of $\mathfrak{g}_{(t)}$ is denoted by $\mathfrak{g}_{(t)}^{\xi}$. 
Each Lie algebra $\mathfrak{g}_{(t)}$ has its own singular set $\mathfrak{g}_{(t), \text { sing }}^{*}=\mathfrak{g}^{*} \backslash \mathfrak{g}_{(t) \text {,reg }}^{*}$, which is regarded as a subset of $\mathfrak{g}^{*}$. If $\pi_{t}$ is the Poisson tensor of the bracket $\{,\}_{t}$, then

$$
\mathfrak{g}_{(t), \text { sing }}^{*}=\left\{\xi \in \mathfrak{g}^{*} \mid \operatorname{rk} \pi_{t}(\xi)<\mathrm{rk} \pi_{t}\right\}
$$

which is the union of the coadjoint $G_{(t)}$-orbits in $\mathfrak{g}^{*}$ having a non-maximal dimension. For simplicity, we write $\mathfrak{g}_{\infty, \text { sing }}^{*}$ or $\mathfrak{g}_{\infty, \text { reg }}^{*}$ in place of $\mathfrak{g}_{(\infty) \text {,sing }}^{*}$ or $\mathfrak{g}_{(\infty) \text {,reg }}^{*}$.

Proposition 3.1. The closure of $\bigcup_{t \neq 0, \infty} \mathfrak{g}_{(t), \text { sing }}^{*}$ in $\mathfrak{g}^{*}$ is a subset of codimension at least 2 .

Proof. Let $\xi=\xi_{0}+\xi_{1}+\cdots+\xi_{m-1} \in \mathfrak{g}^{*}$, where $\xi_{i} \in \mathfrak{g}_{i}^{*}$. Using Proposition 2.1(i) and the dual representation $\varphi^{*}: \mathbb{k}^{\star} \rightarrow \mathrm{GL}\left(\mathfrak{g}^{*}\right), s \mapsto \varphi_{s}^{*}$, one readily verifies that $\xi \in \mathfrak{g}_{\text {sing }}^{*}=\mathfrak{g}_{(1), \text { sing }}^{*}$ if and only if $\varphi_{s}^{*}(\xi) \in \mathfrak{g}_{(s), \text { sing }}^{*}$. Therefore,

$$
\bigcup_{t \neq 0, \infty} \mathfrak{g}_{(t), \text { sing }}^{*}=\bigcup_{s \in \mathbb{k}^{\star}} \varphi_{s}^{*}\left(\mathfrak{g}_{\text {sing }}^{*}\right)=\left\{\xi_{0}+s^{-1} \xi_{1}+\cdots+s^{1-m} \xi_{m-1} \mid \xi \in \mathfrak{g}_{\text {sing }}^{*}, s \in \mathbb{k}^{\star}\right\} .
$$

Since codim $\mathfrak{g}_{\text {sing }}^{*}=3$, the closure of $\bigcup_{t \neq 0, \infty} \mathfrak{g}_{(t) \text {,sing }}^{*}$ is a subset of $\mathfrak{g}^{*}$ of codimension at least 2 .

In order to compute tr.deg $z$, we have to elaborate on some relevant properties of the limit Lie algebras $\mathfrak{g}_{(\infty)}$ and $\mathfrak{g}_{(0)}$.

\subsection{Properties of $\mathfrak{g}_{(\infty)}$}

By Proposition $2.3, \mathfrak{g}_{(\infty)}$ is a nilpotent $\mathbb{N}$-graded Lie algebra, hence $G_{(\infty)}$ is a unipotent algebraic group. Recall also that the subspace $\mathfrak{g}_{0}$ belongs to the centre of $\mathfrak{g}_{(\infty)}$.

Theorem 3.2. For any $\vartheta \in \operatorname{Aut}(\mathfrak{g})$ of finite order, one has the equality ind $\mathfrak{g}_{(\infty)}=$ $\operatorname{dim} \mathfrak{g}_{0}+\mathrm{rk} \mathfrak{g}-\mathrm{rkg}_{0}$.

Proof. (1) For $\mu \in \mathfrak{g}_{0}^{*} \subset \mathfrak{g}_{(\infty)}^{*}$, the stabiliser $\mathfrak{g}_{(\infty)}^{\mu}$ is also $\mathbb{N}$-graded. Furthermore, if $\eta \in \mathfrak{g}_{j}$ with $1 \leqslant j \leqslant m-1$, then $\varkappa\left(\mu,\left[\eta, \mathfrak{g}_{m-j}\right]_{\infty}\right)=0$ if and only if $[\eta, \mu]=0$. Hence

$$
\mathfrak{g}_{(\infty)}^{\mu}=\mathfrak{g}^{\mu}+\mathfrak{g}_{0}
$$

where $\mathfrak{g}^{\mu}$ is the usual stabiliser of $\mu \in \mathfrak{g}^{*}$ (with respect to the initial Lie algebra structure on $\mathfrak{g}$ ). As is well known, the reductive subalgebra $\mathfrak{g}_{0}=\mathfrak{g}^{\vartheta}$ contains regular semisimple elements of $\mathfrak{g}$, see, e.g., [Ka83, §8.8]. These elements form a dense open subset of $\mathfrak{g}_{0}$, which is denoted by $\Omega_{0}$. If $h \in \Omega_{0}$, then $\mathfrak{g}^{h}$ is a Cartan subalgebra of $\mathfrak{g}$ and $\mathfrak{g}^{h} \cap \mathfrak{g}_{0}$ is a Cartan subalgebra of $\mathfrak{g}_{0}$. Using the identification of $\mathfrak{g}_{0}$ and $\mathfrak{g}_{0}^{*}$, we may think of the subset $\Omega_{0}^{*}$ of "regular semisimple" elements of $\mathfrak{g}_{0}^{*} \subset \mathfrak{g}_{(\infty)}^{*}$. For $\mu \in \Omega_{0}^{*}$, it follows from (3.1) that $\operatorname{dim} \mathfrak{g}_{(\infty)}^{\mu}=\operatorname{dim} \mathfrak{g}_{0}+\operatorname{rk} \mathfrak{g}-\operatorname{rk} \mathfrak{g}_{0}$ and hence ind $\mathfrak{g}_{(\infty)} \leqslant \operatorname{dim} \mathfrak{g}_{0}+\mathrm{rk} \mathfrak{g}-\mathrm{rk} \mathfrak{g}_{0}$.

(2) Let us prove the opposite inequality. We think of $\mathfrak{g}_{(\infty)}^{*}$ as a graded vector space of the form

$$
\mathfrak{g}_{(\infty)}^{*}=\mathfrak{g}_{0}^{*} \oplus \mathfrak{g}_{1}^{*} \oplus \cdots \oplus \mathfrak{g}_{m-1}^{*}
$$


Write $\operatorname{ad}_{(\infty)}^{*}$ for the coadjoint representation of $\mathfrak{g}_{(\infty)}$. The graded structure of $\mathfrak{g}_{(\infty)}$ described in Proposition 2.3 implies that $\operatorname{ad}_{(\infty)}^{*}$ has the property that

$$
\operatorname{ad}_{(\infty)}^{*}\left(\mathfrak{g}_{j}\right) \cdot \mathfrak{g}_{i}^{*} \subset \mathfrak{g}_{i+m-j}^{*}
$$

Take any $\xi=\sum_{j=0}^{m-1} \xi_{j} \in \mathfrak{g}_{(\infty)}^{*}$ such that $\xi_{0} \in \Omega_{0}^{*}$. Let $h \in \Omega_{0}$ be the regular semisimple element of $\mathfrak{g}$ corresponding to $\xi_{0}$ under our identifications. Set $\mathfrak{t}=\mathfrak{g}^{h}$. Then $[\mathfrak{g}, h]=\mathfrak{t}^{\perp}$ is the orthogonal complement of $\mathfrak{t}$ with respect to $\varkappa$ and $\mathfrak{g}=$ $[\mathfrak{g}, h] \oplus \mathfrak{t}$. For $\mathfrak{t}_{j}=\mathfrak{t} \cap \mathfrak{g}_{j}$, this implies that $\left[\mathfrak{g}_{j}, h\right]=\mathfrak{g}_{j} \cap \mathfrak{t}_{m-j}^{\perp}$ and $\left[\mathfrak{g}_{j}, h\right] \oplus \mathfrak{t}_{j}=\mathfrak{g}_{j}$. Our goal is to prove that the orbit $G_{(\infty)} \cdot \xi$ contains an element $\gamma$ such that

$$
\operatorname{dim} \mathfrak{g}_{(\infty)}^{\gamma} \geqslant \operatorname{dim} \mathfrak{g}_{0}+\mathrm{rk} \mathfrak{g}-\operatorname{rk} \mathfrak{g}_{0}
$$

We perform this step by step, as follows.

For $\eta \in \mathfrak{g}_{m-1}$, we have $\operatorname{ad}_{(\infty)}^{*}(\eta) \cdot \xi_{0} \in \mathfrak{g}_{1}^{*}$ and

$$
\exp \left(\operatorname{ad}_{(\infty)}^{*}(\eta)\right) \cdot \xi \in \xi_{0}+\left(\xi_{1}+\operatorname{ad}_{(\infty)}^{*}(\eta) \cdot \xi_{0}\right)+\left(\bigoplus_{j \geqslant 2} \mathfrak{g}_{j}^{*}\right)
$$

Since $\operatorname{ad}_{(\infty)}^{*}\left(\mathfrak{g}_{m-1}\right) \cdot \xi_{0}=\mathfrak{g}_{1}^{*} \cap \operatorname{Ann}\left(\mathfrak{t}_{1}\right)$, there is $\eta_{1} \in \mathfrak{g}_{m-1}$ such that

$$
\gamma_{1}:=\xi_{1}+\operatorname{ad}_{(\infty)}^{*}\left(\eta_{1}\right) \cdot \xi_{0} \in \mathfrak{t}_{1}^{*} .
$$

Applying $\exp \left(\operatorname{ad}_{(\infty)}^{*}(\eta)\right)$ with $\eta \in \mathfrak{g}_{m-2}$ to $\exp \left(\operatorname{ad}_{(\infty)}^{*}\left(\eta_{1}\right)\right) \xi=\xi_{0}+\gamma_{1}+\xi_{2}^{\prime}+\ldots$, we do not affect the summands $\xi_{0}$ and $\gamma_{1}$. In doing so, we can replace $\xi_{2}^{\prime}$ with $\gamma_{2} \in \mathfrak{t}_{2}^{*}$. Continuing in this way, we get in $G_{(\infty)} \cdot \xi$ an element of the form $\gamma=\xi_{0}+\sum_{j=1}^{m-1} \gamma_{j}$ with $\gamma_{j} \in \mathfrak{t}_{j}^{*}$. It is easily seen that $\mathfrak{t} \subset \mathfrak{g}_{(\infty)}^{\gamma}$, hence

$$
\operatorname{dim} \mathfrak{g}_{(\infty)}^{\gamma} \geqslant \operatorname{dim} \mathfrak{g}_{0}+\operatorname{rk} \mathfrak{g}-\operatorname{rk} \mathfrak{g}_{0},
$$

as required.

Corollary 3.3. One has $\infty \in \mathbb{P}_{\text {reg }}$ if and only if $\operatorname{dim} \mathfrak{g}_{0}=\mathrm{rk} \mathfrak{g}_{0}$, i.e., $\mathfrak{g}_{0}$ is an abelian subalgebra of $\mathfrak{g}$.

Remark 3.4. (1) There is a short proof of the corollary that does not use Theorem 3.2 in full strength. If $\infty \in \mathbb{P}_{\text {reg }}$, then $\mathcal{Z}_{\infty} \subset \mathcal{Z}$. Since $\mathfrak{g}_{0}$ belongs to the centre of $\mathfrak{g}_{(\infty)}$, we have $\mathfrak{g}_{0} \subset \mathcal{Z}_{\infty}$. Hence $\mathfrak{g}_{0}$ has to be abelian in $\mathfrak{g}$. Conversely, if $\mathfrak{g}_{0}$ is abelian, then, for $\mu \in \Omega_{0}^{*}$ in the first part of the proof, we obtain $\operatorname{dim} \mathfrak{g}_{(\infty)}^{\mu}=\operatorname{rk} \mathfrak{g}$. Hence ind $\mathfrak{g}_{(\infty)}=$ rk $\mathfrak{g}$ and $\infty \in \mathbb{P}_{\text {reg. }}$.

(2) By V. G. Kac's classification of elements of finite order in $G$ [Ka83, Chap. 8], if $\mathfrak{g}$ is simple, $\vartheta$ is inner, and $\mathfrak{g}_{0}=\mathfrak{g}^{\vartheta}$ is abelian, then $\operatorname{ord}(\vartheta)$ is at least the Coxeter number of $\mathfrak{g}$. This means that, for many interesting examples with small ord $(\vartheta)$, we have $\infty \in \mathbb{P}_{\text {sing }}$.

Let us check another technical condition, which is required below. Recall from Section 1.1 that $\pi(\gamma)=\hat{\gamma}$ is a skew-symmetric bilinear form on $\mathfrak{g}$ associated with $\gamma \in \mathfrak{g}^{*}$. We then need the rank of the restriction $\left.\pi(\xi)\right|_{V}$, if $\xi \in \mathfrak{g}^{*}$ is generic and $V=\operatorname{ker} \pi_{\infty}(\xi)=\mathfrak{g}_{(\infty)}^{\xi}$. Set $\mathfrak{g}_{>0}=\bigoplus_{j>0} \mathfrak{g}_{j}$ and $\mathfrak{g}_{>0}^{*}=\bigoplus_{j>0} \mathfrak{g}_{j}^{*}$. 
Lemma 3.5. For any $\vartheta$ and generic $\xi \in \mathfrak{g}^{*}$, we have $\operatorname{rk}\left(\left.\pi(\xi)\right|_{V}\right)=\operatorname{dim} V-\operatorname{rk} \mathfrak{g}$. Proof. Take any $\xi \in \mathfrak{g}_{\mathrm{reg}}^{*} \cap \mathfrak{g}_{\infty, \mathrm{reg}}^{*}$. Then $\operatorname{rk}\left(\left.\pi(\xi)\right|_{V}\right) \leqslant \operatorname{dim} V-\operatorname{rk} \mathfrak{g}$ by [PY, Appendix] and $\operatorname{dim} V=\operatorname{dim} \mathfrak{g}_{0}+\mathrm{rk} \mathfrak{g}-\operatorname{rk} \mathfrak{g}_{0}$ by Theorem 3.2. Write $\xi=\xi_{0}+\xi^{\prime}$ with $\xi_{0} \in \mathfrak{g}_{0}^{*}$ and $\xi^{\prime} \in \mathfrak{g}_{>0}^{*}$. Assume also that $\xi_{0} \in\left(\mathfrak{g}_{0}\right)_{\text {reg }}^{*}$. Note that this is an open condition on $\xi$, too.

As $\mathfrak{g}_{0} \subset V$ (cf. Eq. 3.1), one can write $V=\mathfrak{g}_{0} \oplus V^{\prime}$ for some $V^{\prime} \subset \mathfrak{g}_{>0}$. Here we have $\operatorname{dim} V^{\prime}=\operatorname{rk} \mathfrak{g}-\operatorname{rk} \mathfrak{g}_{0}$. Let $\widetilde{V}$ be the kernel of $\left.\pi(\xi)\right|_{V}$. Then $\widetilde{V} \cap \mathfrak{g}_{0} \subset \mathfrak{g}_{0}^{\xi_{0}}$ and

$$
\operatorname{rk} \mathfrak{g}_{0} \geqslant \operatorname{dim}\left(\widetilde{V} \cap \mathfrak{g}_{0}\right) \geqslant \operatorname{dim} \tilde{V}-\operatorname{dim} V^{\prime}=\operatorname{dim} \tilde{V}+\operatorname{rk} \mathfrak{g}_{0}-\operatorname{rk} \mathfrak{g} .
$$

Hence we have $\operatorname{dim} \tilde{V} \leqslant \operatorname{rk} \mathfrak{g}$ and thereby $\operatorname{rk}\left(\left.\pi(\xi)\right|_{V}\right) \geqslant \operatorname{dim} V-\operatorname{rk} \mathfrak{g}$. This settles the claim.

For future use, we record yet another property of $\mathfrak{g}_{(\infty)}$.

Lemma 3.6. If $\xi=\xi_{0}+\xi^{\prime}$, where $\xi^{\prime} \in \mathfrak{g}_{>0}^{*}$, and $\xi_{0} \in \mathfrak{g}_{0}^{*}$ is regular in $\mathfrak{g}$, then $\xi \in \mathfrak{g}_{\infty, \mathrm{reg}}^{*}$.

Proof. As in Proposition 2.1(iv), consider the invertible linear map $\psi_{s}: \mathfrak{g} \rightarrow \mathfrak{g}$ such that $\psi\left(x_{j}\right)=s^{m-j} x_{j}$ for $x_{j} \in \mathfrak{g}_{j}$ and $s \in \mathbb{k}^{\star}$. It follows from Proposition 2.3 that $\psi_{s}$ is an automorphism of the graded Lie algebra $\mathfrak{g}_{(\infty)}$. Let $\psi_{s}^{*}$ denote the induced action on $\mathfrak{g}_{(\infty)}^{*}$, i.e., $\psi_{s}^{*}\left(\xi_{j}\right)=s^{j-m} \xi_{j}$ if $\xi_{j} \in \mathfrak{g}_{j}^{*}$. Then $\operatorname{dim} \mathfrak{g}_{(\infty)}^{\xi}=\operatorname{dim} \mathfrak{g}_{(\infty)}^{\psi_{s}^{*}(\xi)}$ for any $\xi \in \mathfrak{g}^{*}$. Note that $\lim _{s \rightarrow 0} s^{m} \psi_{s}(\xi)=\xi_{0}$. If $\xi_{0}$ is regular in $\mathfrak{g}_{(\infty)}^{*}$, then so is $\xi$. It remains to deal with $\xi_{0}$.

In view of $(3 \cdot 1), \operatorname{dim} \mathfrak{g}_{(\infty)}^{\xi_{0}}=\operatorname{dim} \mathfrak{g}_{0}+\operatorname{dim} \mathfrak{g}^{\xi_{0}}-\operatorname{dim} \mathfrak{g}_{0}^{\xi_{0}}$. Because $\xi_{0}$ is regular in $\mathfrak{g}$, it is also regular in $\mathfrak{g}_{0}$. Thus $\operatorname{dim} \mathfrak{g}_{(\infty)}^{\xi_{0}}=\operatorname{ind} \mathfrak{g}_{(\infty)}$, and we are done.

\subsection{Pencils of skew-symmetric matrices and differentials}

Recall that any $F \in \mathcal{S}(\mathfrak{g})$ is regarded as a polynomial function on $\mathfrak{g}^{*}$. Then $d F$ is the differential of $F$, which is a polynomial mapping from $\mathfrak{g}^{*}$ to $\mathfrak{g}$. If $\gamma \in \mathfrak{g}^{*}$, then $d_{\gamma} F \in \mathfrak{g}$ stands for the value of $d F$ at $\gamma$.

For a subalgebra $\mathcal{A} \subset \mathcal{S}(\mathfrak{g})$ and $\gamma \in \mathfrak{g}^{*}$, set $d_{\gamma} \mathcal{A}=\left\langle d_{\gamma} F \mid F \in \mathcal{A}\right\rangle_{\mathbb{k}}$. Then $\operatorname{tr} . \operatorname{deg} \mathcal{A}=\max _{\gamma \in \mathfrak{q}^{*}} \operatorname{dim} d_{\gamma} \mathcal{A}$. By the definition of $z$, we have $d_{\gamma} z=\sum_{t \in \mathbb{P}_{\text {reg }}} d_{\gamma} \mathcal{Z}_{t}$. Since $\mathcal{Z}_{t}$ is the centre of $\left(\mathcal{S}(\mathfrak{g}),\{,\}_{t}\right)$, there is the inclusion $d_{\gamma} \mathcal{Z}_{t} \subset \operatorname{ker} \pi_{t}(\gamma)$ for each $t \in \mathbb{P}$ and each $\gamma \in \mathfrak{g}^{*}$.

Let $\left\{H_{1}, \ldots, H_{l}\right\}, l=\mathrm{rk} \mathfrak{g}$, be a set of homogeneous algebraically independent generators of $\mathcal{S}(\mathfrak{g})^{\mathfrak{g}}$. For any $H \in \mathcal{S}(\mathfrak{g})^{\mathfrak{g}}$, we have $d_{\xi} H \in \mathfrak{g}^{\xi}$. The Kostant regularity criterion for $\mathfrak{g}$, see $[\mathrm{K} 63$, Thm. 9], asserts that

$$
\left\langle d_{\xi} H_{j} \mid 1 \leqslant j \leqslant l\right\rangle_{\mathbb{k}}=\mathfrak{g}^{\xi} \text { if and only if } \xi \in \mathfrak{g}_{\mathrm{reg}}^{*} .
$$

Clearly, this criterion applies to semisimple algebras $\mathfrak{g}_{(t)}$ when $t \neq 0, \infty$. That is,

$$
\text { if } t \neq 0, \infty \text {, then } \xi \in \mathfrak{g}_{(t) \text {,reg }}^{*} \Leftrightarrow d_{\xi} \mathcal{Z}_{t}=\operatorname{ker} \pi_{t}(\xi) \Leftrightarrow \operatorname{dim} \operatorname{ker} \pi_{t}(\xi)=\operatorname{rk} \mathfrak{g} \text {. }
$$

As a step towards describing $\mathcal{Z}$, we first consider the smaller algebra

$$
\mathcal{Z}_{\times}=\operatorname{alg}\left\langle\mathcal{Z}_{t} \mid t \in \mathbb{k}^{\star}\right\rangle \subset \mathcal{Z}
$$


An open subset of an algebraic variety is said to be big, if its complement does not contain divisors. By Proposition 3.1, there is a big open subset $U_{\text {sr }} \subset \mathfrak{g}^{*}$ such that $\xi \in \mathfrak{g}_{(t) \text {,reg }}^{*}$ for any $\xi \in U_{\text {sr }}$ and any $t \neq 0, \infty$. Suppose that $\xi \in U_{\text {sr }}$. Then

$$
d_{\xi} z_{\times}=\sum_{t \in \mathbb{k}^{\star}} \operatorname{ker} \pi_{t}(\xi) \subset \sum_{t: \operatorname{rk} \pi_{t}(\xi)=\operatorname{dim} \mathfrak{g}-l} \operatorname{ker} \pi_{t}(\xi)=: L(\xi)
$$

Let us recall a method that provides an upper bound on $\operatorname{dim} L(\xi)$ and thereby on tr.deg $z_{x}$, see [PY20, Sect. 1.1].

Let $\mathcal{P}(\xi)=\left\{c \pi_{t}(\xi) \mid c \in \mathbb{k}, t \in \mathbb{P}\right\}$ be a pencil of skew-symmetric 2-forms on $\mathfrak{g}$, which is spanned by $\pi(\xi)$ and $\pi_{0}(\xi)$. A 2-form in this pencil is said to be regular if $\operatorname{rk}\left(c \pi_{t}(\xi)\right)=\operatorname{dim} \mathfrak{g}-l$. Otherwise, it is singular. Set $U_{\text {srr }}=U_{\mathrm{sr}} \cap \mathfrak{g}_{(0), \text { reg }}^{*} \cap \mathfrak{g}_{\infty, \text { reg }}^{*}$. It is a dense open subset of $\mathfrak{g}^{*}$, which may not be big.

Proposition 3.7. If $\xi \in U_{\text {srr }}$, then $d_{\xi} z_{x}=d_{\xi} z=L(\xi)$.

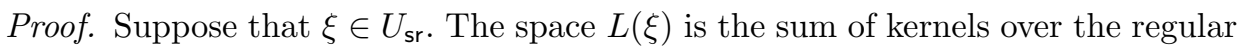
lines in the pencil. Since $\mathbb{P}_{\text {reg }} \backslash \mathbb{k}^{\star}$ is finite, we have $\sum_{t \in \mathbb{k}^{\star}} \operatorname{ker} \pi_{t}(\xi)=L(\xi)$ by $[\mathrm{PY} 08$, Appendix]. Hence $d_{\xi} z_{\times}=L(\xi)$. If $\xi \in U_{\text {srr }}$, then $d_{\xi} z \subset L(\xi)$. Thereby $d_{\xi} z_{\times} \subset$ $d_{\xi} z \subset L(\xi)$, which leads to the equality $d_{\xi} z_{x}=d_{\xi} z$.

Corollary 3.8. For a generic $\xi \in U_{\text {srr }}$, we have $\operatorname{dim} L(\xi)=\operatorname{tr} . \operatorname{deg} z_{\times}=\operatorname{tr} . \operatorname{deg} z$. In particular, $z_{\times} \subset z$ is an algebraic extension.

Although three dimensions in Corollary 3.8 are equal, we do not know yet their exact value. For a given $\xi \in U_{\text {sr }}$, $\operatorname{dim} L(\xi)$ depends on the number of singular lines in $\mathcal{P}(\xi)$.

Proposition 3.9 ([PY20, Prop. 1.5]). If $\xi \in U_{\mathrm{sr}}$, then

(i) $\operatorname{dim} L(\xi)=\boldsymbol{b}(\mathfrak{g})$ if and only if all nonzero 2 -forms in $\mathcal{P}(\xi)$ are regular;

(ii) if $\mathcal{P}(\xi)$ contains a singular line, say $\mathbb{k} \pi_{t^{\prime}}(\xi)$, then $\operatorname{dim} L(\xi) \leqslant l+\frac{1}{2} \mathrm{rk} \pi_{t^{\prime}}(\xi)$;

(iii) furthermore, $\operatorname{dim} L(\xi)=l+\frac{1}{2} \mathrm{rk} \pi_{t^{\prime}}(\xi)$ if and only if

- $\mathbb{k} \pi_{t^{\prime}}(\xi)$ is the only singular line in $\mathcal{P}(\xi)$, and

- $\operatorname{rk}\left(\left.\pi(\xi)\right|_{V}\right)=\operatorname{dim} V-l$ for $V=\operatorname{ker} \pi_{t^{\prime}}(\xi)$.

By Corollary $3.8, z$ is algebraic over $z_{x}$, and it follows from $(2 \cdot 2)$ that $z_{x} \subset$ $\mathcal{S}(\mathfrak{g})^{\mathfrak{g}_{0}}$. Since, being an algebra of invariants, $\mathcal{S}(\mathfrak{g})^{\mathfrak{g}_{0}}$ is an algebraically closed subalgebra of $\mathcal{S}(\mathfrak{g})$, we conclude that

$$
\mathcal{Z} \subset \mathcal{S}(\mathfrak{g})^{\mathfrak{g}_{0}}
$$

By [MY19, Prop. 1.1], if $\mathcal{A}$ is a Poisson-commutative subalgebra of $\mathcal{S}(\mathfrak{g})^{\mathfrak{g}_{0}}$, then

$$
\operatorname{tr} . \operatorname{deg} \mathcal{A} \leqslant \boldsymbol{b}(\mathfrak{g})-\boldsymbol{b}\left(\mathfrak{g}_{0}\right)+\operatorname{rk} \mathfrak{g}_{0}=: \boldsymbol{b}(\mathfrak{g}, \vartheta)
$$

Note that $\boldsymbol{b}(\mathfrak{g}, \vartheta) \leqslant \boldsymbol{b}(\mathfrak{g})$ and the equality occurs if and only if $\mathfrak{g}_{0}$ is abelian. The most interesting case is that in which the upper bound in $(3 \cdot 7)$ is attained for $\mathcal{A}=z$. 
Theorem 3.10. If $\vartheta$ has the property that ind $\mathfrak{g}_{(0)}=$ ind $\mathfrak{g}$, then $\operatorname{tr}$.deg $z=\boldsymbol{b}(\mathfrak{g}, \vartheta)$. Proof. Suppose that $\xi \in U_{\text {srr }}$. Then $\operatorname{rk} \pi_{t}(\xi)=\operatorname{dim} \mathfrak{g}-l$ if and only if $t \in \mathbb{P}_{\text {reg. Since }}$ tr.deg $z \leqslant \boldsymbol{b}(\mathfrak{g}, \vartheta)$, it suffices to show that $\operatorname{dim} L(\xi)=\boldsymbol{b}(\mathfrak{g}, \vartheta)$ whenever $\xi \in U_{\text {srr }}$ is generic.

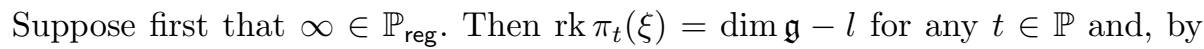
Proposition $3.9(\mathrm{i})$, we obtain $\operatorname{dim} L(\xi)=\boldsymbol{b}(\mathfrak{g})$. And the equality ind $\mathfrak{g}=$ ind $\mathfrak{g}_{(\infty)}$ means that $\mathfrak{g}_{0}$ is abelian, hence $\boldsymbol{b}(\mathfrak{g})=\boldsymbol{b}(\mathfrak{g}, \vartheta)$.

Suppose that $\infty \notin \mathbb{P}_{\text {reg. }}$. Then $\pi_{\infty}(\xi)$ spans the unique singular line in the pencil $c \pi_{t}(\xi)$. By Lemma 3.5, there is a dense open subset $U \subset \mathfrak{g}^{*}$ such that, for any $\eta \in U$ and $V=\operatorname{ker} \pi_{\infty}(\eta)$, we have $\operatorname{rk}\left(\left.\pi(\eta)\right|_{V}\right)=\operatorname{dim} V-l$. If $\xi \in U_{\text {srr }} \cap U$, then conditions (ii) and (iii) of Proposition 3.9 are satisfied for $\pi_{t}(\xi)$. Thus, in this case, $\operatorname{dim} L(\xi)=l+\frac{1}{2} \mathrm{rk} \pi_{\infty}=l+\frac{1}{2}\left(\operatorname{dim} \mathfrak{g}-\operatorname{dim} \mathfrak{g}_{0}-l+\operatorname{rk} \mathfrak{g}_{0}\right)=\boldsymbol{b}(\mathfrak{g})-\boldsymbol{b}\left(\mathfrak{g}_{0}\right)+\operatorname{rk} \mathfrak{g}_{0}=\boldsymbol{b}(\mathfrak{g}, \vartheta)$, since $\operatorname{rk} \pi_{\infty}(\xi)=\operatorname{rk} \pi_{\infty}=\operatorname{dim} \mathfrak{g}-\operatorname{ind} \mathfrak{g}_{(\infty)}$ and ind $\mathfrak{g}_{(\infty)}$ is computed in Theorem 3.2 .

Remark. One can readily prove that the inverse implication in Theorem 3.10 is also true. That is, if tr.deg $z=\boldsymbol{b}(\mathfrak{g}, \vartheta)$, then ind $\mathfrak{g}_{(0)}=$ ind $\mathfrak{g}$.

\subsection{Properties of $\mathfrak{g}_{(0)}$}

Study of Lie algebras $\mathfrak{g}_{(0)}$ associated with arbitrary periodic automorphisms of $\mathfrak{g}$ have been initiated in [P09], where they are called cyclic contractions or $\mathbb{Z}_{k^{-}}$ contractions. In [P09], these algebras are denoted by $\mathfrak{g}\langle k\rangle_{0}$, where $k=\operatorname{ord}(\vartheta)$, because of their interpretation as the fixed-point subalgebras of an extension of $\vartheta$ to an automorphism of $k$-th Takiff algebra modelled on $\mathfrak{g}$. (We discuss Takiff algebras in Section 6.)

The case in which ord $(\vartheta) \geqslant 3$ appears to be more difficult than that of involutions. If $\operatorname{ord}(\vartheta)=2$, then ind $\mathfrak{g}_{(0)}=$ ind $\mathfrak{g}$ and $\mathfrak{g}_{(0)}$ has the codim-2 property [P07]. Whereas, for $\operatorname{ord}(\vartheta) \geqslant 3$, it can happen that $\mathfrak{g}_{(0)}$ does not have the codim-2 property, and the equality ind $\mathfrak{g}_{(0)}=$ ind $\mathfrak{g}$ is only known under certain constraints.

In order to state some sufficient conditions, we first recall several results of E. B. Vinberg [Vi76]. Associated with $\mathbb{Z}_{m}$-grading $\mathfrak{g}=\bigoplus_{i \in \mathbb{Z}_{m}} \mathfrak{g}_{i}$, one has the linear action of $G_{0}$ on $\mathfrak{g}_{1}$. Then

- the algebra of invariants $\mathbb{k}\left[\mathfrak{g}_{1}\right]^{G_{0}}$ is a polynomial (free) algebra;

- the morphism $\pi: \mathfrak{g}_{1} \rightarrow \mathfrak{g}_{1} / / G_{0}=\operatorname{Spec}\left(\mathbb{k}\left[\mathfrak{g}_{1}\right]^{G_{0}}\right)$ is flat and surjective;

- $\pi^{-1} \pi(0)=\mathcal{N} \cap \mathfrak{g}_{1}$ and each fibre of $\pi$ contains finitely many $G_{0}$-orbits.

(It is worth mentioning that this is only a tiny fraction of fundamental results obtained in that great paper.) The fibre $\pi^{-1} \pi(0)$ is customarily called the nullcone (with respect to the $G_{0}$-action on $\mathfrak{g}_{1}$ ) and we denote it by $\mathcal{N}_{1}$.

Definition 3.11. Following [P09], we say that

(1) $\vartheta$ is $\mathcal{S}$-regular, if $\mathfrak{g}_{1}$ contains a regular semisimple element of $\mathfrak{g}$;

(2) $\vartheta$ is $\mathcal{N}$-regular, if $\mathfrak{g}_{1}$ (i.e., $\mathcal{N}_{1}$ ) contains a regular nilpotent element of $\mathfrak{g}$;

(3) $\vartheta$ is very $\mathcal{N}$-regular, if each irreducible component of $\mathcal{N}_{1}$ contains a regular nilpotent element of $\mathfrak{g}$.

If $\operatorname{ord}(\vartheta)=2$, then it follows from [KR71] that properties (1)-(3) are equivalent. But this is not always the case if $\operatorname{ord}(\vartheta) \geqslant 3$. It can happen that $\vartheta$ is $\mathcal{S}$-regular, but 
not $\mathcal{N}$-regular; and vice versa. It can also happen that $\mathcal{N}_{1}$ is reducible and some irreducible components of $\mathcal{N}_{1}$ are not reduced (in the scheme-theoretic sense). Examples of automorphisms of order $\geqslant 3$ such that good properties of Definition 3.11 hold are given in Examples 5.9 and 5.10 in [P09]. The following assertion provides sufficient conditions for some good properties of $\mathfrak{g}_{(0)}$ to hold.

Theorem 3.12 ([P09, Sect. 5]). Let $\vartheta \in \operatorname{Aut}(\mathfrak{g})$ be of finite order.

- If $\mathfrak{g}_{1} \cap \mathfrak{g}_{\text {reg }} \neq \varnothing$, then ind $\mathfrak{g}_{(0)}=$ ind $\mathfrak{g}$;

- If $\vartheta$ is both $\mathcal{S}$-regular and very $\mathcal{N}$-regular, then

- $\mathfrak{g}_{(0)}$ has the codim-2-property;

- $\mathcal{Z}_{0}$ is is freely generated by $H_{1}^{\bullet}, \ldots, H_{l}^{\bullet}, l=\operatorname{rkg}$, where $\left\{H_{1}, \ldots, H_{l}\right\}$ is any set of homogeneous generators of $\mathcal{S}(\mathfrak{g})^{\mathfrak{g}}=\mathcal{Z}_{1}$ that consists of $\vartheta$ eigenvectors.

The assumptions of Theorem 3.12 are not always satisfied, and the property of being "very $\mathcal{N}$-regular" is difficult to check directly. Some methods for handling these properties and related examples can be found in Section 5 in [P09].

There is a nice special case, where all properties of Definition 3.11 hold and Theorem 3.12 applies. Namely, let $\mathfrak{g}$ be the direct sum of $m$ copies of a semisimple Lie algebra $\mathfrak{h}$ and $\vartheta$ a cyclic permutation of the summands; hence $\operatorname{ord}(\vartheta)=m$. Here we obtain a complete description of the Poisson-commutative subalgebra $Z$, see Section 6.

\section{On algebraically independent generators}

If it is known that tr.deg $z=\boldsymbol{b}(\mathfrak{g}, \vartheta)$, then it becomes a meaningful task to compute the minimal number of generators of $z$ or $z_{x}$.

Let $\left\{H_{1}, \ldots, H_{l}\right\}$ be a set of homogeneous algebraically independent generators of $\mathcal{S}(\mathfrak{g})^{\mathfrak{g}}$ and $d_{i}=\operatorname{deg} H_{i}$. Then $\sum_{i=1}^{l} d_{i}=\boldsymbol{b}(\mathfrak{g})$. Recall from Sections 1.2 and 2.1 that associated with $\vartheta$, we have the polynomial homomorphism $\varphi: \mathbb{k}^{\star} \rightarrow \operatorname{GL}(\mathfrak{g})$ and its extension to invertible linear transformations of $\mathcal{S}^{j}(\mathfrak{g})$ for all $j$. Therefore, each $H_{j}$ decomposes as $H_{j}=\sum_{i \geqslant 0} H_{j, i}$, where $\varphi_{s}\left(H_{j}\right)=\sum_{i \geqslant 0} s^{i} H_{j, i}$. The polynomials $H_{j, i}$ are called bi-homogeneous components of $H_{j}$. By definition, the $\varphi$-degree of $H_{j, i}$ is $i$, also denoted by $\operatorname{deg}_{\varphi} H_{j, i}$.

Let $H_{j}^{\bullet}$ be the nonzero bi-homogeneous component of $H_{j}$ with maximal $\varphi$ degree. Then $\operatorname{deg}_{\varphi} H_{j}=\operatorname{deg}_{\varphi} H_{j}^{\bullet}$ and we set $d_{j}^{\bullet}=\operatorname{deg}_{\varphi} H_{j}^{\bullet}$. By Proposition 2.1(i), $\mathcal{Z}_{t}$ is generated by $\varphi_{s}^{-1}\left(H_{1}\right), \ldots, \varphi_{s}^{-1}\left(H_{l}\right)$, where $t=s^{m} \in \mathbb{k}^{\star}$. By the standard argument with the Vandermonde determinant, we then conclude that $z_{\times}$is generated by all bi-homogeneous components of $H_{1}, \ldots, H_{l}$, i.e.,

$$
z_{\times}=\operatorname{alg}\left\langle H_{j, i} \mid 1 \leqslant j \leqslant l, 0 \leqslant i \leqslant d_{j}^{\bullet}\right\rangle .
$$

Definition 4.1. Let us say that $H_{1}, \ldots, H_{l}$ is a good generating system in $\mathcal{S}(\mathfrak{g})^{\mathfrak{g}}$ (g.g.s. for short) for $\vartheta$, if $H_{1}^{\bullet}, \ldots, H_{l}^{\bullet}$ are algebraically independent. Then we also say that $\vartheta$ admits a g.g.s.

The property of being 'good' really depends on a generating system. For instance, for $\mathfrak{g}=\mathfrak{s l}_{n}$ and $m=2$, the coefficients of the characteristic polynomial of $A \in \mathfrak{s l}_{n}$ 
yield a g.g.s., while the polynomials $\operatorname{tr}\left(A^{i}\right), i=2, \ldots, n$ do not provide a g.g.s., see [P07].

The importance of g.g.s. is manifestly seen in the following fundamental result.

Theorem 4.2 ([Y14, Thm.3.8]). Let $H_{1}, \ldots, H_{l}$ be an arbitrary set of homogeneous algebraically independent generators of $\mathcal{S}(\mathfrak{g})^{\mathfrak{g}}$. Then

(i) $\sum_{j=1}^{l} \operatorname{deg}_{\varphi} H_{j} \geqslant \sum_{j=1}^{m-1} j \operatorname{dim} \mathfrak{g}_{j}=: D_{\vartheta}$;

(ii) $H_{1}, \ldots, H_{l}$ is a g.g.s. if and only if $\sum_{j=1}^{l} \operatorname{deg}_{\varphi} H_{j}=D_{\vartheta}$;

(iii) if $\mathfrak{g}_{(0)}$ has the codim-2 property, ind $\mathfrak{g}_{(0)}=l$, and $H_{1}, \ldots, H_{l}$ is a g.g.s., then $\mathcal{Z}_{0}=\mathcal{S}\left(\mathfrak{g}_{(0)}\right)^{\mathfrak{g}_{(0)}}$ is a polynomial algebra freely generated by $H_{1}^{\bullet}, \ldots, H_{l}^{\bullet}$.

Recall that $\operatorname{dim} \mathfrak{g}_{j}=\operatorname{dim} \mathfrak{g}_{m-j}$ for any $0<j \leqslant m-1$. Hence

$$
D_{\vartheta}=\sum_{j=1}^{m-1} j \operatorname{dim} \mathfrak{g}_{j}=\frac{1}{2} \sum_{j=1}^{m-1} m \operatorname{dim} \mathfrak{g}_{j}=\frac{m}{2}\left(\operatorname{dim} \mathfrak{g}-\operatorname{dim} \mathfrak{g}_{0}\right)
$$

Not every $i \in\left\{0,1, \ldots, d_{j}^{\bullet}\right\}$ provides a nonzero bi-homogeneous component $H_{j, i}$. To make this precise, we first consider the case of inner automorphisms, which is technically easier.

Theorem 4.3. Suppose that $\vartheta \in \operatorname{Aut}(\mathfrak{g})$ is inner and admits a g.g.s. Let $H_{1}, \ldots, H_{l}$ be such a g.g.s. in $\mathcal{S}(\mathfrak{g})^{\mathfrak{g}}$ and $d_{j}^{\bullet}=\operatorname{deg}_{\varphi} H_{j}$. Then

(i) each $d_{j}^{\bullet} \in m \mathbb{Z}$ and $H_{j, i} \neq 0$ only if $i \in m \mathbb{Z}$ and $0 \leqslant i \leqslant d_{j}^{\bullet}$;

(ii) if ind $\mathfrak{g}_{(0)}=l$, then $H_{j, i} \neq 0$ for every $i \in m \mathbb{Z}$ with $0 \leqslant i \leqslant d_{j}^{\bullet}$ and the polynomials $\left\{H_{j, i} \mid j=1, \ldots, l ; \& i=0, m, \ldots, d_{j}^{\bullet}\right\}$ are algebraically independent (and generate $z_{\times}$).

Proof. (i) Since $\vartheta$ is inner, $\operatorname{rk} \mathfrak{g}=\operatorname{rk} \mathfrak{g}_{0}$ and $\vartheta\left(H_{j}\right)=H_{j}$ for all $j$. On the other hand, $\vartheta\left(H_{j, i}\right)=\zeta^{i} H_{j, i}$. This proves everything.

(ii) It follows from (i) that the number of nonzero bi-homogeneous components of $H_{j}$ is at most $\left(d_{j}^{\bullet} / m\right)+1$. Hence the total number of nonzero bi-homogeneous components of all $H_{j}$ is at most

$\sum_{j=1}^{l}\left(\frac{d_{j}^{\bullet}}{m}+1\right)=\frac{D_{\vartheta}}{m}+l=\frac{1}{2}\left(\operatorname{dim} \mathfrak{g}-\operatorname{dim} \mathfrak{g}_{0}\right)+l=\boldsymbol{b}(\mathfrak{g})-\boldsymbol{b}\left(\mathfrak{g}_{0}\right)+\operatorname{rk} \mathfrak{g}_{0}=\boldsymbol{b}(\mathfrak{g}, \vartheta)$,

where the equality rk $\mathfrak{g}=\mathrm{rk} \mathfrak{g}_{0}$ is used. On the other hand, the hypothesis ind $\mathfrak{g}_{(0)}=$ $l$ guarantees us that tr.deg$z_{x}=\operatorname{tr} \cdot \operatorname{deg} z_{x}=\boldsymbol{b}(\mathfrak{g}, \vartheta)$, cf. Theorem 3.10 and Corollary 3.8. As the bi-homogeneous components $\left\{H_{j, i}\right\}$ generate $z_{\times}(4 \cdot 1)$, all $H_{j, i}$ with $i / m \in \mathbb{Z}$ must be nonzero and algebraically independent.

With extra technicalities, the same idea works for the arbitrary automorphisms as well. Let $\vartheta \in \operatorname{Aut}(\mathfrak{g})$ be an arbitrary automorphism of order $m$. Since $\vartheta$ acts on $\mathcal{S}(\mathfrak{g})^{\mathfrak{g}}$, there is a set of homogeneous generators $\left\{H_{j}\right\} \subset \mathcal{S}(\mathfrak{g})^{\mathfrak{g}}$ such that each $H_{j}$ is an eigenvector of $\vartheta$, i.e., $\vartheta\left(H_{j}\right)=\zeta^{r_{j}} H_{j}$ for some $r_{j} \in \mathbb{Z}$. However, we need a set of free generators that simultaneously is a g.g.s. and consists of $\vartheta$-eigenvectors. For $m=2$, this is proved in [PY, Lem. 3.4]. The following is an adaptation of that argument to any $m \geqslant 2$. 
Lemma 4.4. If $\vartheta$ admits a g.g.s., then there is also a g.g.s. that consists of $\vartheta$ eigenvectors.

Proof. Let $H_{1}, \ldots, H_{l}$ be a g.g.s., hence $\sum_{j=1}^{l} \operatorname{deg}_{\varphi} H_{j}=D_{\vartheta}$ in view of Theorem 4.2.

Let $\mathcal{A}_{+}$be the ideal in $\mathcal{S}(\mathfrak{g})^{\mathfrak{g}}$ generated by all homogeneous invariants of positive degree. Then $\mathcal{A}:=\mathcal{A}_{+} / \mathcal{A}_{+}^{2}$ is a finite-dimensional $\mathbb{k}$-vector space. If $H \in \mathcal{A}_{+}$, then $\bar{H}:=H+\mathcal{A}_{+}^{2} \in \mathcal{A}$. As is well known, $F_{1}, \ldots, F_{m}$ is a generating system for $\mathcal{S}(\mathfrak{g})^{\mathfrak{g}}$ if and only if the $\mathbb{k}$-linear span of $\bar{F}_{1}, \ldots, \bar{F}_{m}$ is the whole of $\mathcal{A}$. In our situation, $\operatorname{dim}_{\mathbb{k}} \mathcal{A}=l$ and $\mathcal{A}=\left\langle\bar{H}_{1}, \ldots, \bar{H}_{l}\right\rangle_{\mathbb{k}}$.

If $H_{i}$ is not a $\vartheta$-eigenvector, i.e., $\vartheta\left(H_{i}\right) \notin \mathbb{k} H_{i}$, then we consider the (nonminimal) generating set

$$
\left\{H_{1}, \ldots, H_{i-1}, H_{i+1}, \ldots, H_{l}\right\} \bigcup\left\{H_{i}^{[k]}=\sum_{j=0}^{m-1} \zeta^{j k} \vartheta^{j}\left(H_{i}\right) \mid 0 \leqslant k<m\right\}
$$

for $\mathcal{S}(\mathfrak{g})^{\mathfrak{g}}$ that includes $l+m-1$ polynomials. Since $\bar{H}_{1}, \ldots, \bar{H}_{i-1}, \bar{H}_{i+1}, \ldots, \bar{H}_{l}$ are linearly independent in $\mathcal{A}$, one can pick out a suitable $H_{i}^{[k]}$ such that

$$
\bar{H}_{1}, \ldots, \bar{H}_{i-1}, H_{i}^{[k]}, \bar{H}_{i+1}, \ldots, \bar{H}_{l}
$$

is again a minimal generating set. Let us demonstrate that there is a unique suitable choice of $H_{i}^{[k]}$, and this yields a g.g.s. as well. Recall that $d_{j}^{\bullet}=\operatorname{deg}_{\varphi} H_{j}^{\bullet}=\operatorname{deg}_{\varphi} H_{j}$.

Clearly $\operatorname{deg}_{\varphi} H_{i}^{[k]} \leqslant d_{i}^{\bullet}$ for each $k$. Since $\sum_{j=0}^{m-1} \zeta^{j k^{\prime}}=0$ if $1 \leqslant k^{\prime} \leqslant m-1$, we see that $\operatorname{deg}_{\varphi} H_{i}^{[k]}=d_{i}^{\bullet}$ if $\left(k+d_{i}^{\bullet}\right) \in m \mathbb{Z}$ and $\operatorname{deg}_{\varphi} H_{i}^{[k]}<d_{i}^{\bullet}$ for all other $k$. If $\operatorname{deg}_{\varphi} H_{i}^{[k]}<d_{i}^{\bullet}$, then the sum of $\varphi$-degrees for $H_{1}, \ldots, H_{i-1}, H_{i}^{[k]}, H_{i+1}, \ldots, H_{l}$ is less than $D_{\vartheta}$. By Theorem 4.2, this means that such choice of $H_{i}^{[k]}$ in place of $H_{i}$ provides a set of algebraically dependent polynomials. Thus, the only right choice is to take $k$ such that $k+d_{i}^{\bullet} \equiv 0(\bmod m)$, when Theorem 4.2 also guarantees us that we obtain a g.g.s.

The procedure reduces the number of generators that are not $\vartheta$-eigenvectors, and we eventually obtain a g.g.s. that consists of $\vartheta$-eigenvectors.

Let $\left\{H_{1}, \ldots, H_{l}\right\} \subset \mathcal{S}(\mathfrak{g})^{\mathfrak{g}}$ be a generating set consisting of $\vartheta$-eigenvectors. Then $\vartheta\left(H_{i}\right)=\zeta^{r_{i}} H_{i}$ with $0 \leqslant r_{i}<m$. The integers $\left\{r_{i}\right\}$ depend only on the connected component of Aut $(\mathfrak{g})$ that contains $\vartheta$, and if $a$ is the order of $\vartheta$ in $\operatorname{Aut}(\mathfrak{g}) / \operatorname{Int}(\mathfrak{g})$, then $\zeta^{a r_{i}}=1$. Therefore, if $\mathfrak{g}$ is simple, then $\zeta^{r_{i}}= \pm 1$ for all types but $\mathrm{D}_{4}$.

Lemma 4.5. For any $\vartheta \in \operatorname{Aut}(\mathfrak{g})$ of order $m$, we have

(1) $\vartheta\left(H_{j}\right)=H_{j}$ if and only if $d_{j}^{\bullet} \in m \mathbb{Z}$;

(2) $\sum_{j=1}^{l} r_{j}=\frac{1}{2} m\left(\mathrm{rk} \mathfrak{g}-\mathrm{rkg}_{0}\right)$;

(3) $\operatorname{rk} \mathfrak{g}_{0}=\#\left\{j \mid \vartheta\left(H_{j}\right)=H_{j}\right\}$.

Proof. (1) The proof is similar to that of Theorem 4.3(i).

(2) Recall that $\mathfrak{g}_{0}$ always contains regular semisimple elements of $\mathfrak{g}[\mathrm{Ka} 83, \S 8.8]$. Therefore, if $\mathfrak{t}_{0}$ is a Cartan subalgebra of $\mathfrak{g}_{0}$, then $\mathfrak{t}=\mathfrak{z}_{\mathfrak{g}}\left(\mathfrak{t}_{0}\right)$ is a $\vartheta$-stable Cartan subalgebra of $\mathfrak{g}$. Since $\left.\varkappa\right|_{\mathfrak{t}}$ is non-degenerate, $\operatorname{dim}\left(\mathfrak{t} \cap \mathfrak{g}_{j}\right)=\operatorname{dim}\left(\mathfrak{t} \cap \mathfrak{g}_{m-j}\right)$ for all $j<m$. 
Let us apply Kostant's regularity criterion (cf. (3·3)) to some $x \in \mathfrak{t}_{0} \cap \mathfrak{g}_{\text {reg }}$. According to this criterion, $\left\langle d_{x} H_{j} \mid 1 \leqslant j \leqslant l\right\rangle_{\mathbb{k}}=\mathfrak{t}$. Here $d_{x} H_{j} \in \mathfrak{t} \cap \mathfrak{g}_{i}$ if and only if $r_{j}=i$. Therefore

$$
\sum_{j=1}^{l} r_{j}=\sum_{i=1}^{m-1} i \operatorname{dim}\left(\mathfrak{t} \cap \mathfrak{g}_{i}\right)=\frac{1}{2} m\left(\operatorname{dim} \mathfrak{t}-\operatorname{dim} \mathfrak{t}_{0}\right) .
$$

(3) To prove this, it suffices to notice that $d_{x} H_{j} \in \mathfrak{g}_{0}$ if and only if we have $\vartheta\left(H_{j}\right)=H_{j}$.

Now, we can prove our main result on $z_{x}$.

Theorem 4.6. Suppose that $\vartheta \in \operatorname{Aut}(\mathfrak{g})$ admits a g.g.s. and ind $\mathfrak{g}_{(0)}=$ rk $\mathfrak{g}$. Then

(i) $Z_{\times}$is a polynomial Poisson-commutative subalgebra of $\mathcal{S}(\mathfrak{g})^{\mathfrak{g}_{0}}$ having the maximal transcendence degree.

(ii) More precisely, if $H_{1}, \ldots, H_{l}$ is a g.g.s. that consists of $\vartheta$-eigenvectors, then $Z_{\times}$is freely generated by the nonzero bi-homogeneous components of all $\left\{H_{j}\right\}$.

Proof. We already know that $z_{\times}$is Poisson-commutative and tr.deg $z_{\times}=\boldsymbol{b}(\mathfrak{g}, \vartheta)$ is the maximal possible (Theorem 3.10). Hence we have to prove the polynomiality and (ii).

Recall that $\left\{H_{j}\right\}$ is a g.g.s. if and only if $\sum_{j=1}^{l} d_{j}^{\bullet}=D_{\vartheta}=m\left(\operatorname{dim} \mathfrak{g}-\operatorname{dim} \mathfrak{g}_{0}\right) / 2$. If $\vartheta\left(H_{j}\right)=H_{j}$, i.e., $d_{j}^{\bullet} \in m \mathbb{Z}$, then $H_{j}$ has at most $\left(d_{j}^{\bullet} / m\right)+1$ nonzero bihomogeneous components, as in the case of Theorem 4.3. In general, if $\vartheta\left(H_{j}\right)=$ $\zeta^{r_{j}} H_{j}$, then $H_{j, i}$ can be nonzero only if $i \equiv r_{j}(\bmod m)$. Therefore, $d_{j}^{\bullet} \equiv r_{j}$ $(\bmod m)$ and $H_{j}$ has at most $1+\left(d_{j}^{\bullet}-r_{j}\right) / m$ nonzero bi-homogeneous components. Using Lemma 4.5, we see that the total number of all nonzero bi-homogeneous components is at most

$$
\begin{aligned}
\sum_{j=l}^{l}\left(\frac{d_{j}^{\bullet}-r_{j}}{m}+1\right) & =\left(\sum_{j=1}^{l} \frac{d_{j}^{\bullet}}{m}\right)-\left(\sum_{j=1}^{l} \frac{r_{j}}{m}\right)+l \\
& =\frac{D_{\vartheta}}{m}-\frac{l-\operatorname{rk} \mathfrak{g}_{0}}{2}+l=\boldsymbol{b}(\mathfrak{g})-\boldsymbol{b}\left(\mathfrak{g}_{0}\right)+\operatorname{rk} \mathfrak{g}_{0}=\boldsymbol{b}(\mathfrak{g}, \vartheta) .
\end{aligned}
$$

On the other hand, it follows from Eq. (4.1) that the total number of bi-homogeneous components of $H_{1}, \ldots, H_{l}$ is at least $\boldsymbol{b}(\mathfrak{g}, \vartheta)$. Therefore, all admissible bihomogeneous components must be nonzero and algebraically independent.

A precise relationship between $z$ and $z_{\times}$depends on further properties of $\vartheta$. Two complementary assertions are given below.

Corollary 4.7. In addition to the hypotheses of Theorem 4.6 , suppose that $\mathfrak{g}_{(0)}$ has the codim-2 property and $\mathfrak{g}_{0}=\mathfrak{g}^{\vartheta}$ is not abelian. Then $\mathcal{Z}=\mathcal{Z}_{\times}$is the polynomial algebra freely generated by all nonzero bi-homogeneous components $H_{j, i}$.

Proof. In this case, $0 \in \mathbb{P}_{\text {reg }}$ and it follows from Theorem 4.2 (iii) that $\mathcal{Z}_{0}$ is equal to $\mathbb{k}\left[H_{1}^{\bullet}, \ldots, H_{l}^{\bullet}\right]$, which is contained in $z_{\times}$. On the other hand, $\infty \in \mathbb{P}_{\text {sing }}$ (Corollary 3.3), hence $\mathcal{Z}_{\infty}$ is not required for $z$. Thus, $z=z_{\times}$. 
Corollary 4.8. In addition to the hypotheses of Theorem 4.6, suppose that $\mathfrak{g}_{(0)}$ has the codim-2 property, $\vartheta$ is inner, and $\mathfrak{g}_{0}=\mathfrak{g}^{\vartheta}$ is abelian. Then $\mathcal{Z}_{\infty}=\mathcal{S}\left(\mathfrak{g}_{0}\right)$ and $z=\operatorname{alg}\left\langle\mathcal{Z}_{\times}, \mathfrak{g}_{0}\right\rangle$ is a polynomial algebra.

Proof. Recall that the subspace $\mathfrak{g}_{0}$ lies in the centre of $\mathfrak{g}_{(\infty)}$, hence $\mathcal{S}\left(\mathfrak{g}_{0}\right) \subset \mathcal{Z}_{\infty}$. If $\vartheta$ is inner, then ind $\mathfrak{g}_{(\infty)}=\operatorname{dim} \mathfrak{g}_{0}=\operatorname{rkg}\left(\right.$ Theorem 3.2). Hence $\mathcal{S}\left(\mathfrak{g}_{0}\right) \subset \mathcal{Z}_{\infty}$ is an algebraic extension. Since $\mathcal{S}\left(\mathfrak{g}_{0}\right)$ is an algebraically closed subalgebra of $\mathcal{S}(\mathfrak{g})$, it coincides with $\mathcal{Z}_{\infty}$. As in the previous corollary, we have $\mathcal{Z}_{0} \subset \mathcal{Z}_{\times}$. Therefore,

$$
z=\operatorname{alg}\left\langle\mathcal{Z}_{\times}, \mathcal{Z}_{\infty}\right\rangle=\operatorname{alg}\left\langle\mathcal{Z}_{\times}, \mathfrak{g}_{0}\right\rangle
$$

Among the algebraically independent generators of $z_{\times}$, one has $l$ nonzero functions $H_{j, 0} \in \mathcal{S}\left(\mathfrak{g}_{0}\right), j=1, \ldots, l$. (Note that $d_{j}^{\bullet} \in m \mathbb{Z}$ for each $j$, since $\vartheta$ is inner.) Hence the passage from $z_{\times}$to $z$ merely means that we have to replace $\left\{H_{j, 0}\right\}$ with a basis for $\mathfrak{g}_{0}$.

Remark 4.9. If $\vartheta$ does not admit a g.g.s., then $H_{1}^{\bullet}, \ldots, H_{l}^{\bullet}$ are algebraically dependent. Since $\left\{H_{i}^{\bullet}\right\}$ are certain bi-homogeneous components, this means that the number of all nonzero bi-homogeneous components of $\left\{H_{j}\right\}$ is larger than tr.deg $Z$. Moreover, the case of involutions $(m=2)$ shows that then the algebra $\mathcal{Z}_{0}=$ $\mathcal{S}\left(\mathfrak{g}_{(0)}\right)^{\mathfrak{g}_{(0)}}$, which is contained in $z$, is not polynomial, see [Y17, Sect. 5]. Therefore, it would be unwise to expect really good properties of $z_{\times}$or $z$ without the presence of a g.g.s.

\section{On the maximality problem}

Since $z \subset \mathcal{S}(\mathfrak{g})^{\mathfrak{g}_{0}}$ by $(3 \cdot 6)$, the algebra $\tilde{z}:=\operatorname{alg}\left\langle z, \mathcal{S}\left(\mathfrak{g}_{0}\right)^{\mathfrak{g}_{0}}\right\rangle$ is still Poissoncommutative. It was proved in $[\mathrm{PY}]$ that if $\vartheta$ is an involution admitting a g.g.s., then $\tilde{z}$ is a maximal Poisson-commutative subalgebra of $\mathcal{S}(\mathfrak{g})^{\mathfrak{g}_{0}}$. For $m \geqslant 3$, the same problem becomes more difficult, and we obtain only partial results in this section. Our line of argument employs properties of graded polynomial algebras.

Let $F_{1}, \ldots, F_{N} \in \mathbb{k}\left[\left\{x_{i}\right\}\right]=\mathbb{k}\left[\mathbb{A}^{n}\right]$ be algebraically independent homogeneous polynomials. Each differential $d F_{i}$ is a regular 1 -form on $\mathbb{A}^{n}$ with polynomial coefficients. Then

$$
d F_{1} \wedge \cdots \wedge d F_{N}=\boldsymbol{f} R
$$

where $\boldsymbol{f} \in \mathbb{k}\left[\mathbb{A}^{n}\right]$ and $R$ is a regular differential $N$-form that is nonzero on a big open subset. Note that $\boldsymbol{f}$ is defined uniquely up to multiplication with scalars. Let $\mathcal{F}=\mathbb{k}\left[\left\{F_{j}\right\}\right]$ be the subalgebra of $\mathbb{k}\left[\mathbb{A}^{n}\right]$ generated by the polynomials $F_{j}$.

Theorem 5.1 ([PPY, Thm. 1.1]). If $\boldsymbol{f}=1$, then $\mathcal{F}$ is an algebraically closed subalgebra of $\mathbb{k}\left[\mathbb{A}^{n}\right]$, i.e., if $H \in \mathbb{k}\left[\mathbb{A}^{n}\right]$ is algebraic over the field $\mathbb{k}\left(F_{1}, \ldots, F_{N}\right)$, then $H \in \mathcal{F}$.

Consider the following conditions on the Lie algebra $\mathfrak{g}_{(0)}$ :

$\left(\diamond_{1}\right)$ ind $\mathfrak{g}_{(0)}=\mathrm{rk} \mathfrak{g}=l$, i.e., $0 \in \mathbb{P}_{\text {reg }}$;

$\left(\diamond_{2}\right) \quad \mathcal{Z}_{0}$ is a polynomial ring generated by $H_{i}^{\bullet}$ with $1 \leqslant i \leqslant l$;

$\left(\diamond_{3}\right) \operatorname{dim} \mathfrak{g}_{(0) \text {,sing }}^{*} \leqslant \operatorname{dim} \mathfrak{g}-2$, i.e., $\mathfrak{g}_{(0)}$ has the codim-2 property;

$\left(\diamond_{4}\right)$ either $\mathfrak{g}_{0}$ is non-abelian or $\mathfrak{g}_{0}$ is abelian and $\vartheta$ is inner. 
These conditions imply that $Z$ is a polynomial algebra, see Corollary 4.7 and 4.8 . Moreover, the following is true.

Proposition 5.2. If conditions $\left(\diamond_{1}\right)-\left(\diamond_{4}\right)$ are satisfied, then $\tilde{z}$ is a polynomial algebra, too.

Proof. By Lemma 4.5, we have $H_{j, 0} \neq 0$ if and only if $\vartheta\left(H_{j}\right)=H_{j}$, and the number of such $j$ 's equals rk $\mathfrak{g}_{0}$. Then we have $H_{j, 0} \in \mathcal{S}\left(\mathfrak{g}_{0}\right)^{\mathfrak{g}_{0}}$. In the passage from $z$ to $\tilde{z}$, these nonzero generators $H_{j, 0}$ are replaced with the basic symmetric invariants of $\mathfrak{g}_{0}$.

Lemma 5.3. Assume that $\left(\nabla_{1}\right)$ and $\left(\diamond_{3}\right)$ hold. Suppose that there is a divisor $D \subset \mathfrak{g}^{*}$ such that $\operatorname{dim} d_{\eta} z_{\times}<\boldsymbol{b}(\mathfrak{g}, \vartheta)$ for any $\eta \in D$. Then $D=Y \times \mathfrak{g}_{>0}^{*}$, where $Y \subset \mathfrak{g}_{0}^{*} \cap \mathfrak{g}_{\text {sing }}^{*}$ is a $G_{0}$-stable conical divisor in $\mathfrak{g}_{0}^{*}$.

Proof. By Proposition 3.1, there is a big open subset $U_{\text {sr }} \subset \mathfrak{g}^{*}$ such that

$$
U_{\mathrm{sr}} \subset \bigcap_{t \neq 0, \infty} \mathfrak{g}_{(t), \mathrm{reg}}^{*}
$$

By $\left(\diamond_{3}\right), \mathfrak{g}_{(0) \text {,reg }}^{*}$ is also a big open subset of $\mathfrak{g}^{*}$. Hence $\tilde{U}:=D \cap U_{\mathrm{sr}} \cap \mathfrak{g}_{(0) \text {,reg }}^{*}$ is open and dense in $D$. Take any $\eta \in \tilde{U}$. If we write $\eta=\eta_{0}+\eta^{\prime}$ with $\eta \in \mathfrak{g}_{0}^{*}$ and $\eta^{\prime} \in \mathfrak{g}_{>0}^{*}$, then we may also assume that $\eta_{0} \in\left(\mathfrak{g}_{0}\right)_{\mathrm{reg}}^{*}$. Assume that $\eta_{0} \in \mathfrak{g}_{\mathrm{reg}}^{*}$. Then $\eta \in \mathfrak{g}_{\infty, \text { reg }}^{*}$ by Lemma 3.6. Thus, in that case, $\eta \in U_{\text {srr }}$. Moreover, such $\eta$ is generic in the sense of Lemma 3.5 and the conclusion of that lemma holds for it. Arguing as in the proof of Theorem 3.10 and using $(3 \cdot 5)$, we obtain that $\operatorname{dim} L(\eta)=\boldsymbol{b}(\mathfrak{g}, \vartheta)$, a contradiction!

Therefore we must have $\eta_{0} \in \mathfrak{g}_{\text {sing }}^{*}$ for a generic $\eta \in D$ and hence for any $\eta \in D$. Since $\mathfrak{g}_{0}^{*} \cap \mathfrak{g}_{\text {sing }}^{*}$ is a proper subset of $\mathfrak{g}_{0}^{*}$, the divisor $D$ is indeed of the form $Y \times \mathfrak{g}_{>0}^{*}$, where $Y \subset \mathfrak{g}_{0}^{*} \cap \mathfrak{g}_{\text {sing }}^{*}$.

The algebra $Z_{\times}$consists of $G_{0}$-invariants and the group $G_{0}$ is connected. Thereby each irreducible component of the subset $\left\{\gamma \in \mathfrak{g}^{*} \mid \operatorname{dim} d_{\gamma} z_{\times}<\boldsymbol{b}(\mathfrak{g}, \vartheta)\right\}$ is $G_{0}$-stable. In particular, $D$, and hence $Y$ as well, is $G_{0}$-stable. Since $z_{\times}$is a homogeneous subalgebra, the divisor $Y \subset \mathfrak{g}_{0}^{*}$ is conical.

Theorem 5.4. Suppose that $\left(\nabla_{1}\right)$, $\left(\diamond_{2}\right)$, and $\left(\diamond_{3}\right)$ hold. If $\mathfrak{g}_{0} \cap \mathfrak{g}_{\mathrm{sing}}^{*}$ does not contain divisors, then $\mathcal{S}\left(\mathfrak{g}_{0}\right)^{\mathfrak{g}_{0}} \subset \mathcal{Z}_{\times}$and $\boldsymbol{z}_{\times}=\mathcal{z}=\tilde{z}$ is a maximal Poissoncommutative subalgebra of $\mathcal{S}(\mathfrak{g})^{\mathfrak{g}_{0}}$.

Proof. We know that $z_{\times}$is a polynomial algebra (Theorem 4.6) and tr.deg $z_{\times}=$ $\boldsymbol{b}(\mathfrak{g}, \vartheta)$ (Theorem 3.10). Suppose that $\boldsymbol{Z}_{\times} \subset \mathcal{A} \subset \mathcal{S}(\mathfrak{g})^{\mathfrak{g}_{0}}$ and $\mathcal{A}$ is Poisson-commutative. Then we have $\operatorname{tr} \cdot \operatorname{deg} \mathcal{A} \leqslant \boldsymbol{b}(\mathfrak{g}, \vartheta)$ by $(3 \cdot 7)$. In view of Lemma 5.3 , the differentials $d F_{i}$ of the algebraically independent generators $F_{i} \in z_{\times}$are linearly independent on a big open subset. Then by Theorem $5.1, z_{\times}$is an algebraically closed subalgebra of $\mathcal{S}(\mathfrak{g})$. In particular, we must have $\mathcal{A}=\mathcal{Z}_{\times}$. This applies to $z$ and $\tilde{z}$ as well.

Remark 5.5. Conditions $\left(\diamond_{1}\right)-\left(\diamond_{4}\right)$ are satisfied for involutions $\vartheta$ that have g.g.s.. 
Example 5.6. Theorem 5.4 applies to several outer automorphisms of semisimple Lie algebras, for instance, to $\vartheta \in \operatorname{Aut}(\mathfrak{g})$ of order $2 m$ in case $\mathfrak{g}=\left(\mathfrak{s l}_{2 n}\right)^{m}$ and $\mathfrak{g}_{0}$ is a diagonally embedded $\mathfrak{s p}_{2 n}$, cf. [P09, (3.0)]. Automorphisms of this form are considered in Section 8.

Theorem 3.12 provides a bunch of automorphisms $\vartheta$ with $\operatorname{ord}(\vartheta) \geqslant 3$ such that $\tilde{z}$ is a polynomial algebra, see Examples 5.9 and 5.10 in [P09]. In all these cases, $z \neq \tilde{z}$ and we conjecture that $\tilde{z}$ is a maximal Poisson-commutative subalgebra of $\mathcal{S}(\mathfrak{g})^{\mathfrak{g}_{0}}$.

Summarising our previous considerations, we can say that in order to guarantee some good properties of the Poisson-commutative subalgebras $z_{\times}, \mathcal{Z}$, and $\tilde{z}$, the following properties of $\vartheta \in \operatorname{Aut}(\mathfrak{g})$ and thereby of $\mathfrak{g}_{(0)}$ are needed:

(a) ind $\mathfrak{g}_{(0)}=$ ind $\mathfrak{g}$;

(b) $\mathfrak{g}_{(0)}$ has the codim-2 property;

(c) $\vartheta$ admits a g.g.s.

If $\operatorname{ord}(\vartheta)=2$, then (a) and (b) are always satisfied [P07], and a complete description of involutions admitting a g.g.s. is available [Y17]. In a forthcoming paper, we are going to undertake a thorough substantial investigation of these properties for arbitrary $\vartheta$.

\section{The case of a cyclic permutation}

Let $\mathfrak{h}$ be a simple non-abelian Lie algebra and $\mathfrak{g}=\mathfrak{h}^{m}$ the direct sum of $m \geqslant 2$ copies of $\mathfrak{h}$. Then $l=\operatorname{rk} \mathfrak{g}=m \cdot$ rk $\mathfrak{h}$. Let $\vartheta \in \operatorname{Aut}(\mathfrak{g})$ be a cyclic permutation of the summands of $\mathfrak{g}$. That is,

$$
\vartheta\left(x_{1}, \ldots, x_{m}\right)=\left(x_{m}, x_{1}, \ldots, x_{m-1}\right), x_{i} \in \mathfrak{h} .
$$

Then, for $i=0,1, \ldots, m-1$ and $\zeta=\sqrt[m]{1}$, we have

$$
\mathfrak{g}_{i}=\left\{\left(x, \zeta^{-i} x, \ldots, \zeta^{(1-m) i} x\right) \mid x \in \mathfrak{h}\right\} .
$$

In particular, $\mathfrak{g}_{0}=\Delta_{\mathfrak{h}} \simeq \mathfrak{h}$ is the diagonal and each $\mathfrak{g}_{i}$ is isomorphic to $\mathfrak{h}$ as vector space and as $\mathfrak{g}_{0}$-module. Here the Lie algebra $\mathfrak{g}_{(0)}$ is isomorphic to the truncated current algebra

$$
\mathfrak{h}\langle m\rangle:=\mathfrak{h} \otimes \mathbb{k}[t] /\left(t^{m}\right)=\mathfrak{h}[t] /\left(t^{m}\right),
$$

see [P09, Corollary 3.6]. The isomorphism $\mathfrak{g}_{(0)} \simeq \mathfrak{h}\langle m\rangle$ takes $\mathfrak{g}_{i}$ to the image of $\mathfrak{h} t^{i} \subset \mathfrak{h} \otimes \mathbb{k}[t]$ in $\mathfrak{h}\langle m\rangle$. The Lie algebra $\mathfrak{h}\langle m\rangle$ is also known as a (generalised) Takiff algebra modelled on $\mathfrak{h}$. By [RT92, Thm. 2.8], we have ind $\mathfrak{g}_{(0)}=m \cdot \mathrm{rk} \mathfrak{h}=$ rk $\mathfrak{g}$, i.e., $0 \in \mathbb{P}_{\text {reg. }}$ It then follows from Theorem 3.10 that in this case

$$
\operatorname{tr} \cdot \operatorname{deg} Z=\boldsymbol{b}\left(\mathfrak{h}^{m}, \vartheta\right)=\frac{1}{2}((m-1) \operatorname{dim} \mathfrak{h}+(m+1) \operatorname{rk} \mathfrak{h})=(m-1) \boldsymbol{b}(\mathfrak{h})+\operatorname{rk} \mathfrak{h} .
$$

On the other hand, $\mathfrak{g}_{0}$ is not abelian, hence $\mathbb{P}_{\text {sing }}=\{\infty\}$ and $\mathcal{Z}_{\infty}$ is not required for $\mathcal{z}$. Since $\mathfrak{h}\langle m\rangle$ is $\mathbb{N}$-graded and the zero part is semisimple, the nilpotent radical $\mathfrak{h}\langle m\rangle^{u}$ is equal to $\bigoplus_{i=1}^{m-1} \mathfrak{h} t^{i}$. Comparing this with Proposition 2.3 on the 
graded structure of $\mathfrak{q}_{(\infty)}$, we conclude that here $\mathfrak{g}_{(\infty)} \simeq \mathfrak{h}\langle m+1\rangle^{u}$. Since ind $\mathfrak{g}_{(\infty)}$ is computed for any $\vartheta$ in Theorem 3.2, we obtain a new observation that

$$
\text { ind } \mathfrak{h}\langle m\rangle^{u}=(m-2) \mathrm{rk} \mathfrak{h}+\operatorname{dim} \mathfrak{h} \text {. }
$$

Upon the identification $\mathfrak{g}_{1} \simeq \mathfrak{h}$, see Eq. (6.1), an element $x \in \mathfrak{h}$ is nilpotent (resp. semisimple, regular) in $\mathfrak{h}$ if and only if $\left(x, \zeta^{-i} x, \ldots, \zeta^{(1-m) i} x\right) \in \mathfrak{g}_{1}$ is nilpotent (resp. semisimple, regular) in $\mathfrak{g}$. This also implies that the null-cone $\mathcal{N}_{1}$ is isomorphic to the null-cone of $\mathfrak{h}$. Hence $\mathcal{N}_{1}$ is irreducible. Thus, $\vartheta$ is both $\mathcal{S}$-regular and very $\mathcal{N}$-regular.

Let $\left\{H_{1}, \ldots, H_{l}\right\}$ be s set of homogeneous generators of $\mathcal{S}(\mathfrak{g})^{\mathfrak{g}}$ consisting of $\vartheta$ eigenvectors. Since $\vartheta$ is $\mathcal{S}$-regular and very $\mathcal{N}$-regular, it follows from Theorem 3.12 that $\mathfrak{g}_{(0)}$ has the codim-2-property and $\mathcal{Z}_{0}=\mathbb{k}\left[H_{1}^{\bullet}, \ldots, H_{l}^{\bullet}\right]$, see also [RT92]. The last relation also means that $\left\{H_{j}\right\}$ is a g.g.s. for $\vartheta$.

Theorem 6.1. If $\mathfrak{g}=\mathfrak{h}^{m}$ and $\vartheta$ is a cyclic permutation, then the algebra $\mathfrak{z}=$ $\mathcal{Z}\left(\mathfrak{h}^{m}, \vartheta\right)$ is freely generated by the nonzero bi-homogeneous components $H_{j, i}$ with $1 \leqslant j \leqslant l=m \cdot \mathrm{rk} \mathfrak{h}$. Moreover, $z$ is a maximal Poisson-commutative subalgebra of $\mathcal{S}(\mathfrak{g})^{\mathfrak{g}_{0}}$.

Proof. The above discussion shows that conditions $\left(\nabla_{1}\right)-\left(\nabla_{4}\right)$ hold for $\vartheta$. Hence $z$ is freely generated by the nonzero bi-homogeneous components $H_{j, i}$ by Corollary 4.7.

A point $\xi \in \mathfrak{g}_{0}^{*}$ is regular in $\mathfrak{g}^{*}$ if and only if $\xi$ is regular in $\mathfrak{g}_{0}^{*} \simeq \mathfrak{h}^{*}$. Thereby

$$
\operatorname{dim}\left(\mathfrak{g}_{0}^{*} \cap \mathfrak{g}_{\text {sing }}^{*}\right)=\operatorname{dim} \mathfrak{h}-3
$$

and this intersection does not contain divisors of $\mathfrak{g}_{0}^{*}$. Therefore $z$ is a maximal Poisson-commutative subalgebra of $\mathcal{S}(\mathfrak{g})^{\mathfrak{g}_{0}}$ by Theorem 5.4.

It is not hard to produce a generating set $\left\{H_{j}\right\} \subset \mathcal{S}(\mathfrak{g})^{\mathfrak{g}}$ consisting of $\vartheta$ eigenvectors. Suppose that $\mathrm{rk} \mathfrak{h}=r$ and that $\left\{F_{1}, \ldots, F_{r}\right\}$ is a generating set of $\mathcal{S}(\mathfrak{h})^{\mathfrak{h}} \subset \mathcal{S}(\mathfrak{g})$ for the first copy of $\mathfrak{h}$ in $\mathfrak{g}$. For $0 \leqslant k<m$, set

$$
F_{i}^{[k]}=\frac{1}{m}\left(F_{i}+\zeta^{k} \vartheta\left(F_{i}\right)+\zeta^{2 k} \vartheta^{2}\left(F_{i}\right)+\cdots+\zeta^{k(m-1)} \vartheta^{m-1}\left(F_{i}\right)\right) .
$$

Then $\vartheta\left(F_{i}^{[k]}\right)=\zeta^{-k} F_{i}^{[k]}$. Thus, we can take

$$
\left\{H_{j} \mid 1 \leqslant j \leqslant l\right\}=\left\{F_{i}^{[k]} \mid 1 \leqslant i \leqslant r, 0 \leqslant k<m\right\} .
$$

In Section 7, we will need an explicit description of the bi-homogeneous components $H_{j, i}$, which exploits the polarisation construction of [RT92]. Suppose that each $F_{i}$ is homogeneous and $b_{i}=\operatorname{deg} F_{i}$.

For $\xi \in \mathfrak{h}^{*}$, let $\xi^{(k)} \in \mathfrak{g}^{*}$ be the corresponding element sitting in the $k$-th copy of $\mathfrak{h}^{*}$ in $\mathfrak{g}^{*}$. Then the induced action of $\vartheta$ in $\mathfrak{g}^{*}$ is given by the formula:

$$
\vartheta\left(\xi_{1}^{(1)}+\cdots+\xi_{m-1}^{(m-1)}+\xi_{m}^{(m)}\right)=\xi_{2}^{(1)}+\cdots+\xi_{m}^{(m-1)}+\xi_{1}^{(m)}
$$


Accordingly, $\mathfrak{g}_{j}^{*}=\left\{\xi^{(1)}+\zeta^{j} \xi^{(2)}+\cdots+\zeta^{(m-1) j} \xi^{(m)} \mid \xi \in \mathfrak{h}^{*}\right\}$ and we fix the corresponding isomorphisms $\nu_{j}: \mathfrak{h}^{*} \stackrel{\sim}{\rightarrow} \mathfrak{g}_{j}^{*}$, where

$$
\xi \stackrel{\nu_{j}}{\longmapsto}\left(\xi^{(1)}+\zeta^{j} \xi^{(2)}+\cdots+\zeta^{(m-1) j} \xi^{(m)}\right) \in \mathfrak{g}_{j}^{*}
$$

For $\xi_{0}, \ldots, \xi_{m-1}$ with $\xi_{j} \in \mathfrak{g}_{j}^{*}$, let $\bar{\xi}=\left(\xi_{0}, \ldots, \xi_{m-1}\right)$ be the corresponding element of $\mathfrak{g}^{*}$ and $\xi_{0}+\cdots+\xi_{m-1}$ be an element of $\mathfrak{h}^{*}$. For $s \in \mathbb{k}$, set

$$
\phi_{s}(\bar{\xi})=\left(s^{m-1} \xi_{0}, s^{m-2} \xi_{1}, \ldots, \xi_{m-1}\right)
$$

and define the $\phi$-polarisations $F_{[k]}$ of $F \in \mathcal{S}^{d}(\mathfrak{h})$ in the following way:

$$
\begin{aligned}
F\left(\phi_{s}(\bar{\xi})\right): & =F\left(s^{m-1} \xi_{0}+s^{m-2} \xi_{1}+\ldots+\xi_{m-1}\right) \\
& =: F_{[0]}(\bar{\xi})+s F_{[1]}(\bar{\xi})+\cdots+s^{d(m-1)} F_{[d(m-1)]}(\bar{\xi}) .
\end{aligned}
$$

It is convenient to assume that $F_{[k]}=0$ for $k>d(m-1)$.

Example 6.2. If $x \in \mathfrak{h}$ and $0 \leqslant k<m$, then $x_{[k]}=\frac{1}{m}\left(x, \zeta^{-i} x, \ldots, \zeta^{(1-m) i} x\right) \in \mathfrak{g}_{i}$ with $i=m-1-k$.

According to [RT92], $\mathcal{Z}_{0}$ is freely generated by $\left(F_{i}\right)_{[k]}$ with $1 \leqslant i \leqslant r$ and $0 \leqslant k<m$, where $\mathcal{S}(\mathfrak{h})^{\mathfrak{h}}=\mathbb{k}\left[F_{1}, \ldots, F_{r}\right]$.

Proposition 6.3. We have

$$
\left\{H_{j, i} \neq 0 \mid 1 \leqslant j \leqslant l, 0 \leqslant i\right\}=\left\{\left(F_{i}\right)_{[k]} \mid 1 \leqslant i \leqslant r, 0 \leqslant k \leqslant b_{i}(m-1)\right\},
$$

where $\left(F_{i}\right)_{[k]}$ are the $\phi$-polarisations of $F_{i}$ defined by $(6 \cdot 5)$.

Proof. Now we need to distinguish the first copy $\mathfrak{h}^{(1)} \subset \mathfrak{g}$ of $\mathfrak{h}$ from an abstract $\mathfrak{h}$. Suppose that $F^{(1)} \in \mathcal{S}^{d}\left(\mathfrak{h}^{(1)}\right)$ is the image of $F \in \mathcal{S}^{d}(\mathfrak{h})$ under the tautological isomorphism $\mathcal{S}(\mathfrak{h}) \simeq \mathcal{S}\left(\mathfrak{h}^{(1)}\right)$. The combination of (6.4) and the definition of $\varphi_{s}$, see Section 1.2 , leads to

$$
\varphi_{s}\left(F^{(1)}\right)=F_{[(m-1) d]}+s F_{[(m-1) d-1]}+\ldots+s^{(m-1) d-1} F_{[1]}+s^{(m-1) d} F_{[0]}
$$

for $s \in \mathbb{k}^{\star}$. In this notation, $\vartheta\left(F_{[k]}\right)=\zeta^{-d-k} F_{[k]}$. Next we plug the formula for $\varphi_{s}\left(F^{(1)}\right)$ into $(6 \cdot 3)$ and conclude that each bi-homogeneous component of each $F_{i}^{[k]}$ is a $\phi$-polarisation of $F_{i} \in \mathcal{S}^{b_{i}}(\mathfrak{h})$. By Theorem 6.1 , the total number of the nonzero bi-homogeneous components $\left(F_{i}^{[k]}\right)_{i^{\prime}}$ is

$$
\text { tr.deg } \mathcal{Z}=\boldsymbol{b}(\mathfrak{g}, \vartheta)=(m-1) \cdot \boldsymbol{b}(\mathfrak{h})+r .
$$

Since $\mathfrak{h}$ is semisimple, we have $\boldsymbol{b}(\mathfrak{h})=\sum_{i=1}^{r} b_{i}$. Finally, the total number of $\phi$ polarisations $\left(F_{i}\right)_{[k]}$ equals

$$
r+(m-1) \sum_{i=1}^{r} b_{i}=r+(m-1) \cdot \boldsymbol{b}(\mathfrak{h})=\boldsymbol{b}(\mathfrak{g}, \vartheta) .
$$

Hence the two sets in question coincide. 


\section{Gaudin subalgebras}

Let $\mathfrak{h}$ be the same as in the previous section. The enveloping algebra $\mathfrak{U}\left(t^{-1} \mathfrak{h}\left[t^{-1}\right]\right)$ contains a remarkable commutative subalgebra $\mathfrak{z}(\widehat{\mathfrak{h}})$, which is known as the FeiginFrenkel centre [FF92] (see also [F07]). This subalgebra is defined as the centre of the universal affine vertex algebra associated with the affine Kac-Moody algebra $\widehat{\mathfrak{h}}$ at the critical level. In particular, each element of $\mathfrak{z}(\widehat{\mathfrak{h}})$ is annihilated by the adjoint action of $\mathfrak{h}$. The elements of $\mathfrak{z}(\widehat{\mathfrak{h}})$ give rise to higher Hamiltonians of the Gaudin model, which describes a completely integrable quantum spin chain [FFR].

A Gaudin model consists of $n$ copies of $\mathfrak{h}$ and the Hamiltonians

$$
\mathcal{H}_{k}=\sum_{j \neq k} \frac{\sum_{i=1}^{\operatorname{dim} \mathfrak{h}} x_{i}^{(k)} x_{i}^{(j)}}{z_{k}-z_{j}}, 1 \leqslant k \leqslant n,
$$

where $z_{1}, \ldots, z_{n} \in \mathbb{k}$ are pairwise distinct and $\left\{x_{i}^{(k)} \mid 1 \leqslant i \leqslant \operatorname{dim} \mathfrak{h}\right\}$ is a basis for the $k$-th copy of $\mathfrak{h}$ that is orthonormal with respect to $\varkappa$. Letting $\mathfrak{g}=\mathfrak{h}^{n}$, these Gaudin Hamiltonians $\left\{\mathcal{H}_{k}\right\}$ can be regarded as elements of either $\mathfrak{U}(\mathfrak{h})^{\otimes n} \simeq \mathcal{U}(\mathfrak{g})$ or $\mathcal{S}(\mathfrak{g})$. They commute (and hence Poisson-commute) with each other. Then higher Gaudin Hamiltonians are elements of $\mathcal{U}(\mathfrak{h})^{\otimes n}$ that commute with all $\mathcal{H}_{k}$.

Recall the construction of [FFR] that provides a Gaudin subalgebra $\mathcal{G}$ of $\mathcal{U}(\mathfrak{h})^{\otimes n}$. Set $\widehat{\mathfrak{h}}_{-}=t^{-1} \mathfrak{h}\left[t^{-1}\right]$ and let $\Delta \mathfrak{U}\left(\widehat{\mathfrak{h}}_{-}\right) \simeq \mathfrak{U}\left(\widehat{\mathfrak{h}}_{-}\right)$be the diagonal of $\mathfrak{U}\left(\widehat{\mathfrak{h}}_{-}\right)^{\otimes n}$. Then any vector $\vec{z}=\left(z_{1}, \ldots, z_{n}\right) \in\left(\mathbb{k}^{\star}\right)^{n}$ defines a natural homomorphism

$\rho_{\vec{z}}: \Delta \mathcal{U}\left(\widehat{\mathfrak{h}}_{-}\right) \rightarrow \mathcal{U}(\mathfrak{h})^{\otimes n}$ with $\rho_{\vec{z}}\left(x t^{k}\right)=z_{1}^{k} x^{(1)}+z_{2}^{k} x^{(2)}+\cdots+z_{n}^{k} x^{(n)}$ for $x \in \mathfrak{h}$.

By definition, $\mathcal{G}=\mathcal{G}(\vec{z})$ is the image of $\mathfrak{z}(\widehat{\mathfrak{h}})$ under $\rho_{\vec{z}}$, and one can prove that $\mathcal{G}$ contains $\mathcal{H}_{k}$ for all $k$. Hence $\left[\mathcal{G}(\vec{z}), \mathcal{H}_{k}\right]=0$ for each $k$. One also has $\mathcal{G} \subset \mathcal{U}(\mathfrak{g})^{\Delta \mathfrak{h}}$ by the construction.

Remark 7.1. Gaudin subalgebras have attracted a great deal of attention, see, e.g., $[\mathrm{CFR}]$ and references therein. It is standard to work with complex Lie algebras in this framework. Gaudin algebras are closely related to quantum MF subalgebras [R06] and share some of their properties. In particular, for a generic $\vec{z} \in(\mathbb{R})^{n}$, the action of $\mathcal{G}(\vec{z})$ on an irreducible finite-dimensional $\mathfrak{g}$-module $V\left(\lambda_{1}\right) \otimes \ldots \otimes V\left(\lambda_{n}\right)$ is diagonalisable and has a simple spectrum on the subspace of highest weight vectors with respect to the diagonal $\mathfrak{h} \simeq \Delta_{\mathfrak{h}} \subset \mathfrak{h}^{\oplus n}$ [R18].

The cyclic permutation $\vartheta$ is an automorphism of $\mathfrak{g}$ of order $n$. Let now $\zeta$ be a primitive $n$-th root of unity. Then the $\vartheta$-eigenspace $\mathfrak{g}_{j}$ corresponding to $\zeta^{j}$ is

$$
\mathfrak{g}_{j}=\left\{x^{(1)}+\zeta^{-j} x^{(2)}+\zeta^{-2 j} x^{(3)}+\cdots+\zeta^{j(1-n)} x^{(n)} \mid x \in \mathfrak{h}\right\} \simeq \mathfrak{h} .
$$

Let $\bar{t}$ denote the image of $t^{1-n}$ in $\mathbb{k}\left[t^{-1}\right] /\left(t^{-n}-1\right)$. Then $\mathfrak{h}\left[t^{-1}\right] /\left(t^{-n}-1\right)$ has the canonical $\mathbb{Z}_{n}$-grading $\mathfrak{h} \oplus \mathfrak{h} \bar{t} \oplus \mathfrak{h} \bar{t}^{2} \oplus \cdots \oplus \mathfrak{h} \bar{t}^{n-1}$. Thus, $\mathfrak{h}\left[t^{-1}\right] /\left(t^{-n}-1\right) \simeq \mathfrak{g}$ as a $\mathbb{Z}_{n}$-graded Lie algebra. Fix an isomorphism $\mathfrak{g}_{j} \rightarrow \bar{t}^{j} \mathfrak{h}$ as the projection $\operatorname{pr}_{1}: \mathfrak{g} \rightarrow \mathfrak{h}$ on the first summand of $\mathfrak{g}$ combined with the multiplication by $\bar{t}^{j}$. Now we regard $\mathfrak{g}$ as the quotient of $\mathfrak{h}\left[t^{-1}\right]$.

The above identifications related to $\vartheta$ provide a simpler approach to constructing certain Gaudin subalgebras. 
Proposition 7.2. Take $z_{k}=\zeta^{1-k}$ and consider the corresponding Gaudin subalgebra $\mathcal{G}=\mathcal{G}(\vec{z})$ in $\mathcal{U}(\mathfrak{h})^{\otimes n}$. Then $\mathcal{G}$ coincides with the image of $\mathfrak{z}(\widehat{\mathfrak{h}})$ in the quotient $\mathcal{U}\left(\widehat{\mathfrak{h}}_{-}\right) /\left(t^{-n}-1\right)=\mathcal{U}(\mathfrak{g})$.

Proof. Take $a \in \mathbb{Z}_{>0}$ and write $a=n q+j$ with $1 \leqslant j \leqslant n$. Then for $x t^{-a} \in \mathfrak{h}\left[t^{-1}\right]$, we have

$$
\rho_{\vec{z}}\left(x t^{-a}\right)=x^{(1)}+\zeta^{j} x^{(2)}+\zeta^{2 j} x^{(3)}+\cdots+\zeta^{j(n-1)} x^{(n)} \in \mathfrak{g}_{n-j} .
$$

Therefore $\rho_{\vec{z}}\left(x t^{-a}\right)$ identifies with the image $x \bar{t}^{n-j}$ of $x t^{-a}$ in $\mathfrak{h}\left[t^{-1}\right] /\left(t^{-n}-1\right)$.

By a theorem of Feigin and Frenkel [FF92], $\mathfrak{z}(\widehat{\mathfrak{h}})$ is a polynomial ring in infinitely many variables, with a distinguished set of generators. Set $\tau=-\partial_{t}$. There are algebraically independent elements $S_{1}, \ldots, S_{r} \in \mathfrak{z}(\widehat{\mathfrak{h}})$ such that

$$
\mathfrak{z}(\widehat{\mathfrak{h}})=\mathbb{k}\left[\tau^{k}\left(S_{i}\right) \mid 1 \leqslant i \leqslant r, k \geqslant 0\right]
$$

The symbols $\operatorname{gr}\left(S_{i}\right)$ are homogeneous elements of $\mathcal{S}\left(\mathfrak{h} t^{-1}\right)$ and if $F_{i}=\left.\operatorname{gr}\left(S_{i}\right)\right|_{t=1}$, then $\mathbb{k}\left[F_{1}, \ldots, F_{r}\right]=\mathcal{S}(\mathfrak{h})^{\mathfrak{h}}$. The set $\left\{S_{i}\right\}$ is called a complete set of Segal-Sugawara vectors. Keep the notation $b_{i}=\operatorname{deg} F_{i}$.

Lemma 7.3. For $k \geqslant 0$, let $\boldsymbol{F}_{i, k}$ be the symbol of $\rho_{\vec{z}}\left(\tau^{k}\left(S_{i}\right)\right)$. In the case $z_{k}=\zeta^{1-k}$ for $k=1, \ldots, n$, we have then

$$
\left\langle\boldsymbol{F}_{i, k} \mid 0 \leqslant k \leqslant(n-1) b_{i}\right\rangle_{\mathbb{k}}=\left\langle\left(F_{i}\right)_{[k]} \mid 0 \leqslant k \leqslant(n-1) b_{i}\right\rangle_{\mathbb{k}}
$$

for the $\phi$-polarisations $\left(F_{i}\right)_{[k]}$ related to $\mathfrak{g}^{*}=\bigoplus_{j=0}^{n-1} \mathfrak{g}_{j}^{*}$, cf. (6.5).

Proof. Since $\operatorname{gr}\left(S_{i}\right) \in \mathcal{S}\left(t^{-1} \mathfrak{h}\right)$, we have $\boldsymbol{F}_{i, 0} \in \mathcal{S}\left(\mathfrak{g}_{n-1}\right)$ and $\boldsymbol{F}_{i, 0}=n^{b_{i}}\left(F_{i}\right)_{[0]}$. Assuming $n \geqslant 2$, we can state that $\boldsymbol{F}_{i, 1}=n^{b_{i}}\left(F_{i}\right)_{[1]}$. If $k<n$, then clearly $\frac{1}{k !} \boldsymbol{F}_{i, k}=n^{b_{i}}\left(F_{i}\right)_{[k]}$. More generally, as long as $k \leqslant(n-1) b_{i}$, we have

$$
\frac{1}{k ! n^{b_{i}}} \boldsymbol{F}_{i, k}=\left(F_{i}\right)_{[k]}+\sum_{0<j \leqslant k / n}\left(\begin{array}{c}
b_{i}-j-1 \\
j
\end{array}\right)\left(F_{i}\right)_{[k-j n]},
$$

where the leading term of $\frac{1}{k ! n^{b_{i}}} \boldsymbol{F}_{i, k}$ corresponds to those summands of $\operatorname{gr}\left(\tau^{k}\left(S_{i}\right)\right)$ that belong to $\mathcal{S}\left(\mathfrak{h} t^{-n} \oplus \cdots \oplus \mathfrak{h} t^{-1}\right)$.

Let $z \subset S(\mathfrak{g})$ be the Poisson-commutative subalgebra associated with $\vartheta$.

Theorem 7.4. If $z_{k}=\zeta^{1-k}$ for $k=1, \ldots, n$, then the Gaudin algebra $\mathcal{G}(\vec{z}) \subset$ $\mathcal{U}(\mathfrak{g})$ is a quantisation of $Z$.

Proof. By Theorem 6.1, $z$ is generated by the bi-homogeneous components $H_{j, i}$ with $1 \leqslant j \leqslant l$. Proposition 6.3 provides a description of these components. Then by Lemma 7.3 , we have $\mathcal{Z} \subset \operatorname{gr}(\mathcal{G}(\vec{z}))$. Clearly, $\operatorname{gr}(\mathcal{G}(\vec{z})) \subset \mathcal{S}(\mathfrak{g})^{\mathfrak{g}_{0}}$ is a Poisson-commutative subalgebra. Recall that $z$ is a maximal Poisson-commutative subalgebra of $\mathcal{S}(\mathfrak{g})^{\mathfrak{g}_{0}}$ by Theorem 6.1. Thus $\mathcal{z}=\operatorname{gr}(\mathcal{G}(\vec{z}))$ as required.

Remark 7.5. If $z_{k} \neq z_{j}$ for $k \neq j$, then $\operatorname{tr} \cdot \operatorname{deg} \mathcal{G}(\vec{z})=(n-1) \boldsymbol{b}(\mathfrak{h})+\operatorname{rk} \mathfrak{h}$ by [CFR, Prop. 1]. That is, $\operatorname{tr} \operatorname{deg} \mathcal{G}(\vec{z})=\boldsymbol{b}(\mathfrak{g}, \vartheta)$ is the maximal possible for commutative subalgebras of $\mathcal{U}(\mathfrak{g})^{\mathfrak{g}_{0}}$. Theorem 7.4 shows that one particular Gaudin algebra is actually a maximal commutative subalgebra of $\mathcal{U}(\mathfrak{g})^{\mathfrak{g}_{0}}$. Using a connection with the MF subalgebras established in [R06], we can also prove that any $\operatorname{such} \mathcal{G}(\vec{z})$ is a maximal commutative subalgebra of $\mathcal{U}(\mathfrak{g})^{\mathfrak{g}_{0}}$. 


\section{Fixed-point subalgebras in the infinite-dimensional case}

Let $\mathfrak{h}$ be the same as before. Let now $\vartheta \in$ Aut $(\mathfrak{h})$ be an automorphism of order $m$ and $\zeta=\sqrt[m]{1}$ be primitive. To any such $\vartheta$, one associates a $\vartheta$-twisted loop algebra $\mathfrak{h}\left[t, t^{-1}\right]^{\vartheta}=\left(\mathfrak{h}\left[t, t^{-1}\right]\right)^{\vartheta}$, where $\vartheta(t)=\zeta^{-1} t$ and $\vartheta\left(t^{-1}\right)=\zeta t^{-1}$. If $\vartheta$ is an outer automorphism of $\mathfrak{h}$, then $\mathfrak{h}\left[t, t^{-1}\right]^{\vartheta}$ is a subalgebra of a twisted Kac-Moody algebra, see [Ka83, Chap. 8] for details. Similarly to the case of $\widehat{\mathfrak{h}}_{-}$, we identify $\widehat{\mathfrak{h}}_{-}^{\vartheta}:=\left(\widehat{\mathfrak{h}}_{-}\right)^{\vartheta}$ with $\left(\mathfrak{h}\left[t, t^{-1}\right] / \mathfrak{h}[t]\right)^{\vartheta}$. This leads to an action of $(\mathfrak{h}[t])^{\vartheta}$ on $\widehat{\mathfrak{h}}_{-}^{\vartheta}$ and correspondingly on $\mathcal{S}\left(\widehat{\mathfrak{h}}_{-}^{\vartheta}\right)$. In this section, we consider the invariants of $(\mathfrak{h}[t])^{\vartheta}$ in $\mathcal{S}\left(\widehat{\mathfrak{h}}_{-}^{\vartheta}\right)$, i.e., the subalgebra

$$
z\left(\widehat{\mathfrak{h}}_{-}, \vartheta\right)=\mathcal{S}\left(\widehat{\mathfrak{h}}_{-}^{\vartheta}\right)^{(\mathfrak{h}[t])^{\vartheta}}
$$

which can be thought of as a $\vartheta$-twisted analogue of $\operatorname{gr}(\mathfrak{z}(\widehat{\mathfrak{h}}))$, cf. $(0 \cdot 1)$.

Set $\mathfrak{g}=\mathfrak{h}^{n}$. Let $\tilde{\vartheta} \in \operatorname{Aut}(\mathfrak{g})$ be the composition of $\vartheta$ applied to one copy of $\mathfrak{h}$ only and a cyclic permutation of the summands. Formally speaking,

$$
\tilde{\vartheta}\left(y_{1}^{(1)}+y_{2}^{(2)}+\cdots+y_{n}^{(n)}\right)=y_{n}^{(1)}+\vartheta\left(y_{1}\right)^{(2)}+y_{2}^{(3)}+\cdots+y_{n-1}^{(n)}
$$

for any $y_{1}, \ldots, y_{n} \in \mathfrak{h}$. The order of $\tilde{\vartheta}$ is $N=n m$. Similarly to the case of $\widehat{\mathfrak{h}}_{-}$, there are isomorphisms $\mathfrak{g} \simeq \mathfrak{h}[t]^{\vartheta} /\left(t^{N}-1\right)$ and $\mathfrak{g}_{(0)} \simeq \mathfrak{h}[t]^{\vartheta} /\left(t^{N}\right)$.

Set $\mathfrak{q}=\mathfrak{h}_{(0)}$ transferring the notation of Section 3 to $\mathfrak{h}$. Next we want to understand the connection between the Takiff algebra $\mathfrak{q}\langle n\rangle=\mathfrak{q}[t] /\left(t^{n}\right)$, modelled on $\mathfrak{q}$, and $\mathfrak{g}_{(0)}$. Let $\tilde{\zeta}=\sqrt[N]{1}$ be such that $\zeta=\tilde{\zeta}^{n}$. Set also $\omega=\tilde{\zeta}^{m}$.

Lemma 8.1 (cf. [P09, Sect. 3]).

(i) The Takiff algebra $\mathfrak{q}\langle n\rangle$ is a contraction of $\mathfrak{g}_{(0)}$.

(ii) If ind $\mathfrak{q}=\mathrm{rk} \mathfrak{h}$, then ind $\mathfrak{g}_{(0)}=\mathrm{rk} \mathfrak{g}$.

(iii) If there is a g.g.s. for $\vartheta$, then there is a g.g.s. for $\tilde{\vartheta}$.

(iv) If $\mathfrak{q}$ has the codim-2 property, then so does $\mathfrak{g}_{(0)}$.

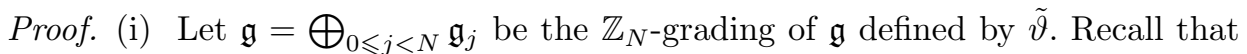
$\mathfrak{g}_{(0)}$ is $\mathbb{N}$-graded and the Lie bracket in $\mathfrak{g}_{(0)}$ is defined by $\left[\mathfrak{g}_{j}, \mathfrak{g}_{j^{\prime}}\right]_{0}=\left[\mathfrak{g}_{j}, \mathfrak{g}_{j^{\prime}}\right] \subset \mathfrak{g}_{j+j^{\prime}}$ if $j+j^{\prime}<N$ and $\left[\mathfrak{g}_{j}, \mathfrak{g}_{j^{\prime}}\right]_{0}=0$ otherwise. The Lie algebra $\mathfrak{q}\langle n\rangle$ is also $\mathbb{N}$-graded and has the same components, however, the Lie bracket is different. Write $j=q m+i$ with $0 \leqslant i<m$ and similarly $j^{\prime}=q^{\prime} m+i^{\prime}$. Then in $\mathfrak{q}\langle n\rangle$, the bracket between $\mathfrak{g}_{j}$ and $\mathfrak{g}_{j^{\prime}}$ is the same as in $\mathfrak{g}_{(0)}$ if $i+i^{\prime}<m$. If $i+i^{\prime} \geqslant m$, then $\mathfrak{g}_{j}$ and $\mathfrak{g}_{j^{\prime}}$ commute in $\mathfrak{q}\langle n\rangle$. This shows that $\mathfrak{q}\langle n\rangle$ is a contraction of $\mathfrak{g}_{(0)}$ corresponding to the following linear map:

$$
\tilde{\psi}_{s}: \mathfrak{g}_{(0)} \rightarrow \mathfrak{g}_{(0)} \text { with } s \in \mathbb{k}^{\star},\left.\tilde{\psi}_{s}\right|_{\mathfrak{g}_{j}}=s^{i} \operatorname{id}_{\mathfrak{g}_{j}} .
$$

(ii) By [RT92, Thm. 2.8(i)], one has ind $\mathfrak{q}\langle n\rangle=n$.ind $\mathfrak{q}$. Therefore, if ind $\mathfrak{q}=\operatorname{rk} \mathfrak{h}$, then ind $\mathfrak{q}\langle n\rangle=n$.ind $\mathfrak{q}=$ rk $\mathfrak{g}$. Because index cannot decrease under contractions, $\operatorname{rk} \mathfrak{g}=$ ind $\mathfrak{g} \leqslant$ ind $\mathfrak{g}_{(0)} \leqslant$ ind $\mathfrak{q}\langle n\rangle=$ rk $\mathfrak{g}$. This settles (ii).

(iii) Let $F_{1}, \ldots, F_{r} \in \mathcal{S}(\mathfrak{h})^{\mathfrak{h}}$ be a g.g.s. for $\vartheta$ such that $\vartheta\left(F_{i}\right)=\zeta^{\ell_{i}} F_{i}$ with $\ell_{i} \in \mathbb{Z}$ for each $i$. Regarding $F_{i}$ as an element of $\mathcal{S}^{b_{i}}\left(\mathfrak{h}^{(1)}\right)$, set

$$
\begin{aligned}
F_{i}^{[k]}=\frac{1}{n}\left(F_{i}+\omega^{k} \tilde{\zeta}^{-\ell_{i}} \tilde{\vartheta}\left(F_{i}\right)\right. & +\omega^{2 k} \tilde{\zeta}^{-2 \ell_{i}} \tilde{\vartheta}^{2}\left(F_{i}\right)+\ldots \\
& \left.+\omega^{(n-1) k} \tilde{\zeta}^{(1-n) \ell_{i}} \tilde{\vartheta}^{n-1}\left(F_{i}\right)\right),
\end{aligned}
$$


cf. (6·3). Here $\tilde{\vartheta}^{n}\left(F_{i}\right)=\vartheta\left(F_{i}\right)=\zeta^{\ell_{i}} F_{i} \in \mathcal{S}\left(\mathfrak{h}^{(1)}\right)$. Furthermore,

$$
\tilde{\vartheta}\left(F_{i}^{[k]}\right)=\omega^{(n-1) k} \tilde{\zeta}^{\ell_{i}} F_{i}^{[k]}, \quad \text { where } \quad \omega^{(n-1) k} \tilde{\zeta}^{\ell_{i}}=\tilde{\zeta}^{\ell_{i}-m k} .
$$

We claim that

$$
\left\{H_{j} \mid 1 \leqslant j \leqslant l\right\}=\left\{F_{i}^{[k]} \mid 1 \leqslant i \leqslant r, 0 \leqslant k<n\right\}
$$

is a g.g.s. for $\tilde{\vartheta}$.

Let $\tilde{\varphi}: \mathbb{k}^{\star} \rightarrow \mathrm{GL}(\mathfrak{g})$ be the polynomial representation such that $\tilde{\varphi}_{s}(x)=s^{j} x$ for $x \in \mathfrak{g}_{j}$. Set $B_{i}^{\bullet}=b_{i}^{\bullet}+b_{i}(n-1) m$. Here $B_{i}^{\bullet} \equiv b_{i}^{\bullet} \equiv \ell_{i}(\bmod m)$. Furthermore, $\operatorname{deg}_{\tilde{\varphi}} F_{i}^{[k]} \leqslant B_{i}^{\bullet}$, because $\tilde{\vartheta}^{v}\left(\mathfrak{h}_{j}^{(1)}\right) \subset \bigoplus_{q=0}^{n-1} \mathfrak{g}_{q m+j}$ for $0 \leqslant j<m$ and $0 \leqslant v$. However, the equality $\operatorname{deg}_{\tilde{\varphi}} F_{i}^{[k]}=B_{i}^{\bullet}$ is possible only if $B_{i}^{\bullet}-\ell_{i}+m k \in N \mathbb{Z}$. If this is not the case for $k$, then $\operatorname{deg}_{\tilde{\varphi}} F_{i}^{[k]} \leqslant B_{i}^{\bullet}-m$. And again, the equality is possible only if $B_{i}^{\bullet}-\ell_{i}+m(k-1) \in N \mathbb{Z}$. Iterating these arguments, we obtain that $\sum_{k=0}^{n-1} \operatorname{deg}_{\tilde{\varphi}} F_{i}^{[k]} \leqslant n b_{i}^{\bullet}+m\left(2 b_{i}-1\right) \frac{n(n-1)}{2}$ for each $1 \leqslant i \leqslant r$. Hence

$$
\sum_{1 \leqslant j \leqslant l} \operatorname{deg}_{\tilde{\varphi}} H_{j} \leqslant n\left(\sum_{i=1}^{r} b_{i}^{\bullet}\right)+m \frac{n(n-1)}{2} \sum_{i=1}^{r}\left(2 b_{i}-1\right)=n D_{\vartheta}+N \frac{n-1}{2} \operatorname{dim} \mathfrak{h}=D_{\tilde{\vartheta}} .
$$

Now the claim follows from Theorem 4.2.

(iv) Suppose that $\mathfrak{q}$ has the codim-2 property, then so does the Takiff algebra $\mathfrak{q}\langle n\rangle$ [RT92, PY20']. Furthermore, if the index does not change under a polynomial contraction, then the dimension of the singular subset can only increase [Y17, (4.1)]. Applying this to $\mathfrak{g}_{(0)}$ and its contraction $\mathfrak{q}\langle n\rangle$, we conclude that $\mathfrak{g}_{(0)}$ has the codim-2 property.

\section{Theorem 8.2.}

(i) If ind $\mathfrak{h}_{(0)}=\mathrm{rk} \mathfrak{h}$, then $\mathcal{z}\left(\widehat{\mathfrak{h}}_{-}, \vartheta\right)$ is a Poisson-commutative subalgebra.

(ii) The algebra $\mathcal{Z}\left(\widehat{\mathfrak{h}}_{-}, \vartheta\right)$ is a polynomial ring (in infinitely many variables) if and only if $\mathcal{S}\left(\mathfrak{g}_{(0)}\right)^{\mathfrak{g}_{(0)}}$ is a polynomial ring for each $n \geqslant 1$ and $\mathfrak{g}=\mathfrak{h}^{n}$ with $\tilde{\vartheta} \in \operatorname{Aut}(\mathfrak{g})$ as above.

Proof. For $N=n m$, set

$$
\mathbb{W}_{N}:=\left(\mathfrak{h} t^{-N} \oplus \ldots \oplus \mathfrak{h} t^{-1}\right)^{\vartheta} \subset \widehat{\mathfrak{h}}_{-}^{\vartheta} .
$$

Then $\mathfrak{h}[t]^{\vartheta}$ acts on $\mathbb{W}_{N}$ via its quotient $\mathfrak{g}_{(0)}$ and $\mathbb{W}_{N} \simeq \mathfrak{g}_{(0)}$ as a $\mathfrak{g}_{(0)}$-module. Therefore there is an isomorphism of commutative algebras $\mathcal{S}\left(\mathbb{W}_{N}\right)^{\mathfrak{h}[t]^{\vartheta}} \simeq \mathcal{S}\left(\mathfrak{g}_{(0)}\right)^{\mathfrak{g}_{(0)}}$.

If $f_{1}, f_{2} \in \mathcal{S}\left(\widehat{\mathfrak{h}}_{-}^{\vartheta}\right)$, then there is $N^{\prime}=n^{\prime} m$ such that $f_{1}, f_{2} \in \mathbb{W}_{N^{\prime}}$. Set $n=2 n^{\prime}$. Then $\left\{f_{1}, f_{2}\right\}=0$ if and only if the images $\bar{f}_{1}, \bar{f}_{2}$ of $f_{1}, f_{2}$ in $\mathcal{S}(\mathfrak{g}) \simeq \mathcal{S}\left(\widehat{\mathfrak{h}}^{\theta}\right) /\left(t^{-N}-1\right)$ Poisson-commute. The $\mathbb{Z}_{N}$-grading of $\mathfrak{g}$ inherited from $\widehat{\mathfrak{h}}_{-}$coincides with the $\mathbb{Z}_{N}$-grading defined by $\tilde{\vartheta}$. If $f_{1}, f_{2} \in z\left(\widehat{\mathfrak{h}}_{-}, \vartheta\right)$, then $\bar{f}_{1}, \bar{f}_{2} \in \mathcal{S}\left(\mathfrak{g}_{(0)}\right)^{\mathfrak{g}_{(0)}}$ by the construction. The assumption ind $\mathfrak{h}_{(0)}=\mathrm{rkh}$ implies that ind $\mathfrak{g}_{(0)}=$ rk $\mathfrak{g}$, see Lemma 8.1. Thus $\mathcal{S}\left(\mathfrak{g}_{(0)}\right)^{\mathfrak{g}_{(0)}}$ is contained in the Poisson-commutative subalgebra $\mathcal{Z}(\mathfrak{g}, \tilde{\vartheta}) \subset \mathcal{S}(\mathfrak{g})$ and hence $\left\{\bar{f}_{1}, \bar{f}_{2}\right\}=0$. 
(ii) The algebra $z\left(\widehat{\mathfrak{h}}_{-}, \vartheta\right)=\lim _{\longrightarrow} \mathcal{S}\left(\mathbb{W}_{n m}\right)^{\mathfrak{h}[t]^{\vartheta}}$ has a direct limit structure. If $\mathcal{Z}\left(\widehat{\mathfrak{h}}_{-}, \vartheta\right)$ is a polynomial ring, then $\mathcal{S}\left(\mathbb{W}_{N}\right)^{\mathfrak{h}[t]^{\vartheta}} \simeq \mathcal{S}\left(\mathfrak{g}_{(0)}\right)^{\mathfrak{g}_{(0)}}$ has to be a polynomial ring for each $n$.

In order to prove the opposite implication, suppose that $\mathcal{S}\left(\mathbb{W}_{N}\right)^{\mathfrak{h}[t]^{\vartheta}}$ is a polynomial ring for each $n$. By a standard argument on graded algebras, see, e.g., the proof of Lemma 4.4, any algebraically independent set of generators of the ring $\mathcal{S}\left(\mathbb{W}_{N}\right)^{\mathfrak{h}[t]^{\vartheta}}$ extends to an algebraically independent set of generators of the ring $\mathcal{S}\left(\mathbb{W}_{N+m}\right)^{\mathfrak{h}[t]^{\vartheta}}$. In the direct limit, one obtains a set of algebraically independent generators of $z\left(\widehat{\mathfrak{h}}_{-}, \vartheta\right)$.

If ind $\mathfrak{h}_{(0)}=\operatorname{rk} \mathfrak{h}$, there is a g.g.s. for $\vartheta$, and $\mathfrak{h}_{(0)}$ has the codim-2 property, then $\mathcal{S}\left(\mathfrak{g}_{(0)}\right)^{\mathfrak{g}_{(0)}}$ is a polynomial ring, see Lemma 8.1 and Theorem 4.2. Set $b_{i, \bullet}=$ $b_{i}(m-1)-b_{i}^{\bullet}$.

Proposition 8.3. If ind $\mathfrak{h}_{(0)}=\mathrm{rk} \mathfrak{h}$, there is a g.g.s. $F_{1}, \ldots, F_{r} \in \mathcal{S}(\mathfrak{h})^{\mathfrak{h}}$ for $\vartheta$, and $\mathfrak{h}_{(0)}$ has the codim-2 property, then $\mathfrak{z}\left(\widehat{\mathfrak{h}}_{-}, \vartheta\right)$ is freely generated by the $\left(t^{-1}\right)$ polarisations $\left(F_{i}\right)_{\left[b_{i, \bullet}+k m\right]}$ with $k \geqslant 0$ related to the subspaces $\left(\left(\mathfrak{h} t^{-j}\right)^{\vartheta}\right)^{*}$.

Proof. First we enlarge on $t^{-1}$-polarisations. For $k \in \mathbb{Z}$, let $\bar{k} \in\{0, \ldots, m-1\}$ be the residue of $k$ modulo $m$. Then $\widehat{\mathfrak{h}}_{-}^{\vartheta}=\bigoplus_{k \leqslant-1} \mathfrak{h}_{\bar{k}} t^{k}$. Let $\phi_{s}:\left(\widehat{\mathfrak{h}}_{-}^{\vartheta}\right)^{*} \rightarrow\left(\widehat{\mathfrak{h}}_{-}^{\vartheta}\right)^{*}$ with $s \in \mathbb{k}^{\star}$ be the linear map multiplying elements of $\left(\mathfrak{h}_{\bar{k}} t^{k}\right)^{*}$ with $s^{-k-1}$. Next we canonically identify $\left(\mathfrak{h}_{\bar{k}} t^{k}\right)^{*}$ with $\mathfrak{h}_{\bar{k}}^{*}$. Then an element of $\left(\widehat{\mathfrak{h}}_{-}^{\vartheta}\right)^{*}$ is a finite sequence $\xi=\left(\xi_{-1}, \xi_{-2}, \ldots, \xi_{-L}\right)$ with $\xi_{k} \in \mathfrak{h}_{\bar{k}}^{*}$. For such a $\xi$, set $|\xi|=\sum_{i=1}^{L} \xi_{-i} \in \mathfrak{h}^{*}$. If $F \in \mathcal{S}(\mathfrak{h})$, then $F\left(\left|\phi_{s}(\xi)\right|\right)=\sum_{k \geqslant 0} s^{k} F_{[k]}(\xi)$, where the sum is actually finite. If $n=1$, then $\mathbb{W}_{m} \simeq \mathfrak{h}_{0}$ and $\left(F_{i}\right)_{\left[b_{i, \bullet}\right]}$ identifies with $F_{i}^{\bullet} \in \mathcal{S}\left(\mathfrak{h}_{(0)}\right)^{\mathfrak{h}_{(0)}}$.

Recall that $\mathcal{Z}\left(\widehat{\mathfrak{h}}_{-}, \vartheta\right)=\lim \mathcal{S}\left(\mathbb{W}_{n m}\right)^{\mathfrak{h}[t]^{\vartheta}}$ and that $\mathcal{S}\left(\mathbb{W}_{N}\right)^{\mathfrak{h}[t]^{\vartheta}} \simeq \mathcal{S}\left(\mathfrak{g}_{(0)}\right)^{\mathfrak{g}_{(0)}}$ for $N=n m$. Now we identify the vector spaces $\mathbb{W}_{N}$ and $\mathfrak{g}$. For $0 \leqslant k<n$, let $F_{i}^{[k]} \in \mathcal{S}(\mathfrak{g})$ be defined by $(8 \cdot 1)$. Then $\mathcal{S}\left(\mathfrak{g}_{(0)}\right)^{\mathfrak{g}_{(0)}}$ is freely generated by the highest components $\left(F_{i}^{[k]}\right)^{\bullet}$ with $1 \leqslant i \leqslant r, 0 \leqslant k<n$. The discussion in the proof of Lemma 8.1(iii) shows that if $B_{i}^{\bullet}-\ell_{i}+m k \in N \mathbb{Z}$, then

$$
\left(F_{i}^{[k]}\right)^{\bullet}=\frac{1}{n^{b_{i}}}\left(F_{i}\right)_{\left[b_{i}, \bullet\right]} \in \mathcal{S}\left(\mathbb{W}_{m}\right)
$$

Analogously to the case of $\vartheta=\mathrm{id}_{\mathfrak{h}}$, cf. Proposition 6.3 , one can see that

$$
\left\{n^{b_{i}}\left(F_{i}^{[k]}\right)^{\bullet} \mid 0 \leqslant k<n\right\}=\left\{\left(F_{i}\right)_{\left[b_{i}, \bullet+k m\right]} \mid 0 \leqslant k<n\right\} .
$$

This completes the proof.

Remark 8.4. Theorem 8.2 emphasises the importance of the equality ind $\mathfrak{h}_{(0)}=$ $\mathrm{rk} \mathfrak{h}$. Let us recall that it holds for the involutions by [P07]. Furthermore, if $\mathfrak{h}$ is a classical Lie algebra and $\vartheta$ is an involution, then there is a g.g.s. for $\vartheta$ and $\mathfrak{h}_{(0)}$ has the codim-2 property [P07], [Y14], i.e., Proposition 8.3 applies. This means that the $\vartheta$-twisted Poisson analogue of the Feigin-Frenkel centre exists for many automorphisms and indicates that probably a $\vartheta$-twisted version of $\mathfrak{z}(\widehat{\mathfrak{h}})$ can be constructed at least for some $\vartheta$. Often both $\vartheta$-twisted objects are expected to be polynomial rings in infinite number of variables. Finally, we mention that results of [PY20'] can be used for the description of $\mathcal{S}\left(\mathfrak{g}_{(0)}\right)^{\mathfrak{g}_{(0)}}$. 


\section{References}

[CFR] A. Chervov, G. Falqui, L. Rybnikov, Limits of Gaudin algebras, quantization of bending flows, Jucys-Murphy elements and Gelfand-Tsetlin bases, Lett. Math. Phys. 91 (2010), no. 2, 129-150.

[DZ05] J.-P. Dufour, N.T. Zung, Poisson Structures and their Normal Forms, Progress in Mathematics, Vol. 242, Birkhäuser Verlag, Basel, 2005.

[FF92] B. Feigin, E. Frenkel, Affine Kac-Moody algebras at the critical level and Gelfand-Dikii algebras, Int. J. Mod. Phys. A7, Suppl. 1A (1992), 197-215.

[FFR] B. Feigin, E. Frenkel, N. Reshetikhin, Gaudin model, Bethe Ansatz and critical level, Comm. Math. Phys. 166 (1994), no. 1, 27-62.

[F07] E. Frenkel, Langlands Correspondence for Loop Groups, Cambridge Studies in Advanced Mathematics, Vol. 103, Cambridge University Press, Cambridge, 2007.

[GZ00] I. M. Gelfand, I. S. Zakharevich, Webs, Lenard schemes, and the local geometry of bi-Hamiltonian Toda and Lax structures, Selecta Math., New Ser. 6 (2000), 131-183.

[Ka83] V. G. Kac, Infinite-dimensional Lie Algebras, Birkhäuser, 1983.

[K63] B. Kostant, Lie group representations on polynomial rings, Amer. J. Math. 85 (1963), 327-404.

[KR71] B. Kostant, S. Rallis, Orbits and representations associated with symmetric spaces, Amer. J. Math. 93 (1971), 753-809.

[MF78] А. С. Мищенко, А. Т. Фоменко, Уравнения Эйлера на конечномерных групnax Лu, Изв. АН СССР. Сер. матем. 42 (1978), вып. 2, 396-415, Engl. transl.: A. S. Mishchenko, A. T. Fomenko, Euler equation on finite-dimensional Lie groups, Math. USSR-Izv. 12 (1978), no. 2, 371-389.

[MY19] A. Molev, O. Yakimova, Quantisation and nilpotent limits of Mishchenko-Fomenko subalgebras, Represent. Theory 23 (2019), 350-378.

[P07] D. Panyushev, On the coadjoint representation of $\mathbb{Z}_{2}$-contractions of reductive Lie algebras, Adv. Math. 213 (2007), 380-404.

[P09] D. Panyushev, Periodic automorphisms of Takiff algebras, contractions, and $\theta$ groups, Transform. Groups 14, no. 2 (2009), 463-482.

[PPY] D. Panyushev, A. Premet, O. Yakimova, On symmetric invariants of centralisers in reductive Lie algebras, J. Algebra 313 (2007), 343-391.

[PY08] D. Panyushev, O. Yakimova, The argument shift method and maximal commutative subalgebras of Poisson algebras, Math. Res. Letters 15 (2008), no. 2, 239249.

[PY20] D. Panyushev, O. Yakimova, Poisson-commutative subalgebras and complete integrability on non-regular coadjoint orbits and flag varieties, Math. Zeitschrift 295 (2020), 101-127.

[PY20'] D. Panyushev, O. Yakimova, Takiff algebras with polynomial rings of symmetric invariants, Transform. Groups 25 (2020), 609-624.

[PY] D. Panyushev, O. Yakimova, Poisson-commutative subalgebras of $\mathcal{S}(\mathfrak{g})$ associated with involutions, Intern. Math. Res. Notices, to appear, DOI:10.1093/imrn/ rnaa041, arXiv: 1809.00350 (2018).

[RT92] M. Raïs, P. Tauvel, Indice et polynômes invariants pour certaines algèbres de Lie, J. reine angew. Math. 425 (1992), 123-140. 
[R06] Л. Г. Рыбников, Метод сдвига инвариантов и модель Годена, Функц. анализ и его прил. 40 (2006), вып. 3, 30-43. Engl. transl.: L. G. Rybnikov, The shift of invariants method and the Gaudin model, Funct. Anal. Appl. 40 (2006), no. 3 188-199.

[R18] L. G. Rybnikov, A proof of the Gaudin Bethe Ansatz conjecture, Intern. Math. Res. Notices 2020 (2020), no. 22, 8766-8785.

[Sp89] T. A. Springer, Aktionen reduktiver Gruppen auf Varietäten, in: Algebraische Transformationsgruppen und Invariantentheorie, DMV-Seminar, Bd. 13, Birkhäuser, Basel, 1989, pp.3-39.

[Vi76] Э. Б. Винберг, Группа Вейля градуированной алгебры Ли, Изв. АН СССР. Cер. Матем. 40 (1976), вып. 3, 488-526. Engl. transl.: E. B. Vinberg, The Weyl group of a graded Lie algebra, Math. USSR-Izv. 10 (1976), 463-495.

[Vi79] Э. Б. Винберг, Классификация однородных нильпотентных әлементов полупростой градуированной алгебры Ли, в сб.: Труды семинара по вект. и тенз. анализу, т. 19, МГУ, М., 1979, с. 155-177. Engl. transl.: E. B. Vinberg, Classification of homogeneous nilpotent elements of a semisimple graded Lie algebra, Selecta Math. Sov. 6 (1987), 15-35.

[Vi90] Э. Б. Винберг, О некоторых коммутативных подалгебрах универсальной обертывающей алгебры, Изв. АН СССР. Сер. матем. 54, вып. 1 (1990), 3 25. Engl transl.: E. B. Vinberg, On certain commutative subalgebras of a universal enveloping algebra, Math. USSR-Izv. 36 (1991), 1-22.

[Lie3] Э. Б. Винберг, В. В. Горбацевич, А. Л. Онищик, Строение групп и алгебр Ли, Итоги науки и техн., Совр. пробл. матем., Фунд. направл., т. 41, Группь Ли и алгебры Ли-3, ВИНИТИ, М., 1990, стр. 5-257. Engl. transl.: V. L. Gorbatsevich, A. L. Onishchik, E. B. Vinberg, Structure of Lie groups and Lie algebras, in: Lie Groups and Lie Algebras III, Encyclopaedia of Mathematical Sciences, Vol. 41, Springer-Verlag, Berlin, 1994, pp. 1-248.

[Y14] O. Yakimova, One-parameter contractions of Lie-Poisson brackets, J. Eur. Math. Soc. 16 (2014), 387-407.

[Y17] O. Yakimova, Symmetric invariants of $\mathbb{Z}_{2}$-contractions and other semi-direct products, Intern. Math. Res. Notices (2017) 2017 (6): 1674-1716.

Funding Information Open Access funding enabled and ogranized by Projeckt DEAL.

Open Access This article is licensed under a Creative Commons Attribution 4.0 International License, which permits use, sharing, adaptation, distribution and reproduction in any medium or format, as long as you give appropriate credit to the original author(s) and the source, provide a link to the Creative Commons licence, and indicate if changes were made. The images or other third party material in this article are included in the article's Creative Commons licence, unless indicated otherwise in a credit line to the material. If material is not included in the article s' Creative Commons licence and your intended use is not permitted by statutory regulation or exceeds the permitted use, you will need to obtain permission directly from the copyright holder. To view a copy of this licence, visit http://creativecommons.org/ licenses/by/4.0/.

Publisher's Note Springer Nature remains neutral with regard to jurisdictional claims in published maps and institutional affiliations. 